\title{
Diagnostics Based on Continuous Scanning LDV Measurements and RASTAR Analysis Method
}

\author{
D. Di Maio ${ }^{1}$ (D) $\cdot$ S. Bruinsma ${ }^{1} \cdot$ T. Tinga ${ }^{1}$
}

Received: 22 April 2020 / Accepted: 20 September 2020 / Published online: 2 October 2020

(C) The Author(s) 2020

\begin{abstract}
This paper presents a technique for rapid diagnostics of structural integrity that exploits spectral sidebands measured by the continuous scanning LDV (CSLDV) measurement method. Structural integrity can be monitored by Operational Deflection Shapes ODSs, which can be quickly measurable at any excitation frequency when a laser continuously scans a vibrating surface. The LDV output time series is an amplitude modulated signal, and its spectral lines are used for recovering polynomial coefficients which build a polynomial function describing a deflection shape. The process of conversion from the time series to a polynomial function can be biased by measurement and analysis uncertainty which eventually will affect the quality of recovered ODS. This paper will show that an effective, rapid and straightforward diagnostics can be yielded by direct analysis of the spectral sidebands without the need to be transformed into polynomial coefficients. The Relative Amplitude of the Sidebands to the Total Amplitude Reference (RASTAR) method is an indicator that exploits the relative amplitude of the sidebands against a datum reference. The relative magnitudes of the sidebands will not change in the absence of any the structural change, and it is independent of the vibration response amplitudes set for the measurements. Such diagnostic method suits maintenance operations of structures which could be quickly scanned by an SLDV system providing an indicative level of structural integrity over the number of duty cycles.
\end{abstract}

Keywords Continuous Scanning LDV $\cdot$ Health Monitoring $\cdot$ Spectral Sidebands

\section{Introduction}

A fundamental part of maintenance technology is monitoring the structural integrity and performance of systems, and by doing so, the remaining life of a component in operating conditions can be estimated. As in recent years, interest surrounding the extension of the life of components has increased to avoid any unexpected and costly downtime. Researchers and companies recognise the importance of maintenance by investing in research and better diagnostic tools. One of the solutions lays in monitoring the structural health (SHM) of mechanical systems, which focuses on the detection of unhealthy dynamic behaviour as damage might occur and propagates in the structure. Looking at the wind power generation,

D. Di Maio

d.dimaio@utwente.nl

1 Applied Mechanics group, ET Faculty, University of Twente, Enschede, Netherlands such as in wind turbines, the SHM is used to detect changes in the system that are the result of damage over time. Nondestructive and vibration-based methods can achieve damage detection. The sensing technology enabling damage detection and characterisation can be either contact or non-contact, depending on the monitoring strategy and system requirements.

Most common contact sensors currently used for SHM are piezoelectric patches, strain gauges and accelerometers, as Chopra reviewed in [1]. Another contact technique that is gaining interest in the field of sensing is the use of fibre optics, where reflecting light gives information on the strain on the component the fibre is attached. Multiple sensors and multiple types of sensors are commonly applied to one component to monitor the condition. These sensors are used in various methods to detect damage. In the research of Johnson et al. [2], a sensor array was used to describe dynamic transmissibility features as indicators of structural damage. The research of Sierra-Perez et al. [3] investigates wind turbine blades for which the authors used fibre optics to detect damage and compared the results to conventional strain gauge methods. It was found that the number of sensors that were needed to perform 
SHM adequately was too high to be viable. Abry et al. [4] proposed a method to detect damage in composites by measuring a change in electrical resistance, which showed that very low damage levels could be detected. However, contact methods are sometimes not viable under operating conditions because they require expensive and/or complicated electronics for transferring the data. Also, determining the location of the sensors is model-based which means that FEA needs to be conducted to find the proper sensor placement.

Many remote sensing methods have been researched, and a selection is presented here, keeping in mind their application in SHM. A widely used and researched method is Digital Image Correlation (DIC). DIC compares multiple digital photographs of a vibrating structure. The pixels in the highresolution photographs are tracked, and after post-processing, a strain-field can be composed. The high contrast and light intensity levels that are required for this technique can be reached in a laboratory environment if adequate lighting is used. In the study, McCormick and Lord [5] presented that the texture of most structures is enough for the tracking software to pick up. However, Avril et al. [6] found that in applications outside the lab, surface treatment is often needed to allow for consistent tracking. This surface conditioning considerably lowers the in situ SHM measurement capabilities of this technique. Detecting damage in composite beams can be done using a thermal camera. When delamination between two plies occurs, the areas rub together and cause heat due to friction. This heat is conducted to the outer surface and can be captured with a thermal optic or infra-red camera. Mian et al. [7] used infra-red surface imaging to detect such delamination. This thermal effect can also be induced by the detection method itself, using sound wave pulses to vibrate crack surfaces, causing them to heat up locally. Currently, this technique is most commonly used for composite materials. The technique can also be applied to metals, but mostly in destructive test environments and near-yield strength tests, as explained by Pastor et al. [8]. The advances made in laser technology allowed for an increase in research of non-contact damage detection methods using laser light. Speckle interferometry is a technique that uses multiple laser beams as well as a camera to extract phase information of a vibrating structure. Jacquot [9] summarises the basic principles behind the widely researched Speckle interferometry technique. The patterns that arise from the interfering laser beams on the rough surface, together with video, yields information on the response of the system to excitation. Guntaka et al. [10] have taken this technique and implemented it for SHM purposes; it can be used for both a pristine and damage case to find differences due to structural degradation. The speckle interferometry technique requires a very expansive and precise set-up. A closely related technique, Holography, uses roughly the same set-up. It is capable of acquiring comprehensive data from the system, as De la Torre et al. [11] reviewed. Holography differs from
Speckle interferometry in that it requires the reconstruction of its 3D complex amplitude [9]. The sterile environment and expansive set-up make these measurement techniques inappropriate for assessing structural health in operating conditions.

A laser-based and eye-safe sensor is the Laser Doppler Vibrometry (LDV). Castellini et al. [12] present the applications of LDV methods, including SHM. In its core, LDV uses a laser beam the light of which is backscattered and collected by a detector. When this is measured (scanned) over time, not only spatial information is obtained, as is the case in Speckle interferometry, but also temporal information. This technique is commonly referred to as Scanning LDV (SLDV). SLDV can be done at discrete points (Stepped SLDV) or with sweeping mirrors, (Continuous SLDV). Both are viable techniques to be used in SHM. However, Stepped SLDV needs several seconds of measuring per discrete measurement point, which is time and data-intensive. Continuous SLDV (CSLDV), scans a line or area over the specimen for several seconds to obtain the information, which makes it a much faster technique, and it measures information from virtually thousands of points in one measurement. Some previous works by Stanbridge et al. [13] and Martarelli [14] explored these techniques in the form of modal testing, where the advantages of CSLDV method are described. CSLDV techniques well-suit structural integrity analysis for damage detection and identification and, in fact, several papers are available to this account. CSLDV techniques were attempted to either cracked beams or plates embedding damages, and these researches can be read in [15-27]. One of the most distinctive features of all the CSLDV papers, cited in here, is the recovery of the ODS(s) as means for further investigation of damage detection and identification. However, there are few papers which aim at exploiting the spectral sidebands directly, as shown in [17, 26, 27], for the purpose of detecting the damage state. The use of spectral sidebands, as straightforward form raw signals, avoids possible bias due to ODS reconstruction, which is based on the recovery of polynomial coefficients. Until this research, the damage identifiers developed in [17, 26, 27] were not robust and depending on the type of damage created to the test structure. The primary objective of this paper is to develop and investigate the use of a novel damage indicator

Table 1 Beam properties

\begin{tabular}{lrlll}
\hline Beam properties & & & Beam Dimensions \\
\cline { 1 - 2 } Material property & Value & & Designation & Length \\
\hline Young's Modulus & $69 \mathrm{MPa}$ & & Length (l) x-direction & $400 \mathrm{~mm}$ \\
Density & $2700 \mathrm{~kg} / \mathrm{m}^{3}$ & & Thickness (t) y-direction & $10 \mathrm{~mm}$ \\
Poisson's Ratio & 0.334 & & Width (w) z-direction & $40 \mathrm{~mm}$ \\
Damping Ratio & 0.004 & & \\
\hline
\end{tabular}


Table 2 Shell element mechanical and dynamic performance

\begin{tabular}{lllll}
\hline & PLANE182 & $\begin{array}{l}\text { SHELL181 } \\
(\text { Full })\end{array}$ & $\begin{array}{l}\text { SHELL181 } \\
\text { (Red.) }\end{array}$ & Analytical \\
\hline $\begin{array}{c}\text { Max. } \delta \\
(\mathrm{m})\end{array}$ & 0.00916 & 0.00914 & 0.00916 & 0.00928 \\
$\begin{array}{c}\text { Error } \omega 1 \\
(\%)\end{array}$ & 0.136 & 0.002 & 0.002 & - \\
$\begin{array}{c}\text { Error } \omega 2 \\
(\%)\end{array}$ & 0.433 & 0.290 & 0.023 & - \\
$\begin{array}{c}\text { Error } \omega 3 \\
(\%)\end{array}$ & 1.653 & 0.727 & 0.726 & - \\
\hline
\end{tabular}

which can assess the structural integrity of a structure by CSLDV measurement methods. This will be possible by analysing the relative amplitude of the spectral sidebands over the total sum of their magnitude between a reference (pristine) and damaged case. This paper will demonstrate this attempt with the aid of a numerical model and the final verification with experimental measurements.

\section{Numerical Investigation}

A numerical model is made in the Finite Element Method (FEM) using ANSYS Mechanical APDL 16.2. For this study, a prismatic, cantilever beam is defined. The beam is clamped at one end and subjected to an excitation force in the ydirection (out-of-plane) at the free end. Standard material properties of aluminium are used, and the beam dimensions are given in Table 1.

Two element types are evaluated, PLANE182 and SHELL181. SHELL181 is a four-node, 3D element with six Degree of Freedom (DOF) if membrane stiffness is included. If not, the element only has 3 DOF (no rotations). Membrane stiffness is used when the main deflection shape is out-ofplane. For this application, in-plane bending of the elements, only membrane stiffness is used. Both full and reduced (red.) integration are considered since multiple elements through the thickness of the beam are chosen. The values from Table 2 show that the SHELL181 element with reduced integration is the best choice since it is closest to the analytical solution. Investigating the deflection shapes revealed a problem with this element. The elements started to behave problematically in a higher frequency range, and the nodal displacements became very unstable. The ODS of one of the problematic frequencies was plotted for all three element configurations. An example frequency of $8500 \mathrm{~Hz}$ is shown in Fig. 1 for all three element configurations.

The elements at the tip on the right-hand side start becoming very unstable both for the PLANE182 and the SHELL181, see Fig. 1. The quality of the deflection shape did not improve with reduced integration, and even rerunning the simulation with more elements. Therefore, the SHELL181 with full integration performed second best was the selected element for the simulations in this work.

\section{Design of the Damage Shale and Location}

Typically damage in Finite Element Analysis can be applied to the model by altering the stiffness properties of the elements. This alteration can be done in several different ways such as deleting damaged elements, reducing the thickness of damaged elements, replacing damaged SHELL elements with spring elements and assigning different material properties to damaged elements. However, it is not advisable to create mesh discontinuities because of the stability of the simulation convergence, where, the thickness reduction of the elements, especially in a two-dimensional model is a better choice also motivated in $[26,28]$. Replacing the SHELL elements with springs is common practice, but it adds modelling complexity when crack paths throughout the beam would be modelled. Assigning different material properties such as lower Young's modulus is less common and could easily alter the dynamic behaviour of the model, as found in [29].
Fig. 1 Behaviour of shell element for the same ODS response

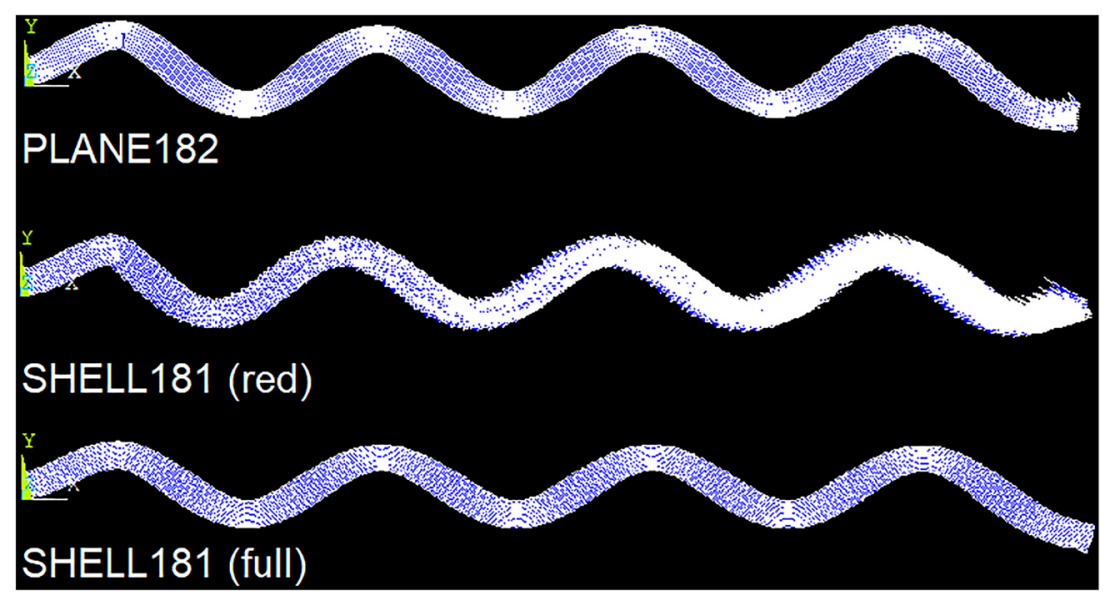


Table 3 Damage types

\begin{tabular}{llcc}
\hline Length & & Transverse & Longitudinal \\
\hline 2 elements & $1 \mathrm{~mm}$ & D10T & D10L \\
5 elements & $2.5 \mathrm{~mm}$ & D25T & D25L \\
10 elements & $5 \mathrm{~mm}$ & D50T & D50L \\
\hline
\end{tabular}

As explained before, multiple different crack paths can be modelled, and in this study, two cuts are made as a transverse crack and as delamination. Aluminium does not develop delamination type of fracture, and it is a practical artefact to investigate the ODS sensitivity to that type of damage. Both damage types were modelled and analysed in three severities: 2 elements ( $10 \%$ thickness), five elements (25\% thickness) and ten elements (50\% thickness). The transverse crack originates from the bottom and is applied at $0.04 \mathrm{~m}$ from the root. The delamination will grow toward the free end from $0.04 \mathrm{~m}$ and is located at $2.5 \mathrm{~mm}$ below the neutral axis. The damage designations for future reference are defined in Table 3.

Figure 2 shows the ANSYS model configuration with examples of damages. The zoomed window displays the possible damage typologies. The red is for the transverse damage, the green is for the delamination, and the purple is for interlaminar damage cracks (not implemented in this study).

\section{Investigation of Damage Behaviour by FEM}

A damage sensitivity analysis of both resonance frequencies and response phase was carried out with the frequency response simulated with the FE model. Figure 3 shows the response frequency simulated for the most severe damage case (50\%) both amplitude and response phase. One can immediately appreciate that the shift in resonances is more apparent for high-frequency modes than the lower ones. However, as both frequency response amplitude and phase shift along the frequency axis, the differential of phase values taken at any fixed (near) resonance frequency between pristine and damage case shows a much larger magnitude than the same differential showed by the resonance frequency change, as presented in Table 4. By looking at damage severities lower than $10 \%$, one can immediately read that the damage state is practically insensitive to the variation of the resonance frequency. Still, it shows high sensitivity to the change of response phase. This sensitivity is explainable by the nature of the response phase, which is steep (depending on damping) at resonance, and thus very sensitive to minimal stiffness variations. This concept can be better appreciated by looking at Figs. 4 and 5, noting that the $\omega_{4}$, as highlighted in Table 4 , is not visible in both plots.

The total strain energy, over the length of the beam, was calculated to understand the underlying behaviour of mode four and on its relatively insensitiveness to the damage. As a matter of comparison, Figs. 6 and 7 show the plots of the ODSs for the second and fourth bending mode for a damage severity equal to $50 \%$. The deflection shape between the pristine and damage case for the second bending is self-evident, whereas no significant change can be appreciated from the fourth deflection shape. By looking at the strain energy for the second bending in Fig. 8 and the fourth bending in Fig. 9, this latter shows nearly null magnitude at the damage location. Therefore, the damage falls at a point where the crack is not subjected to any stress caused by bending motion. Hence, as a result, that mode of vibration is insensitive to such damage.

It was decided to investigate the strain energy calculating ODSs near the resonances and near the anti-resonances. The anti-resonance is the frequency at which the drive point FRF has got a local minimum because of null response at the measurement point. The deflection shape changes from the starting resonance to the following one, and for a cantilever
Fig. 2 Damage shapes and locations

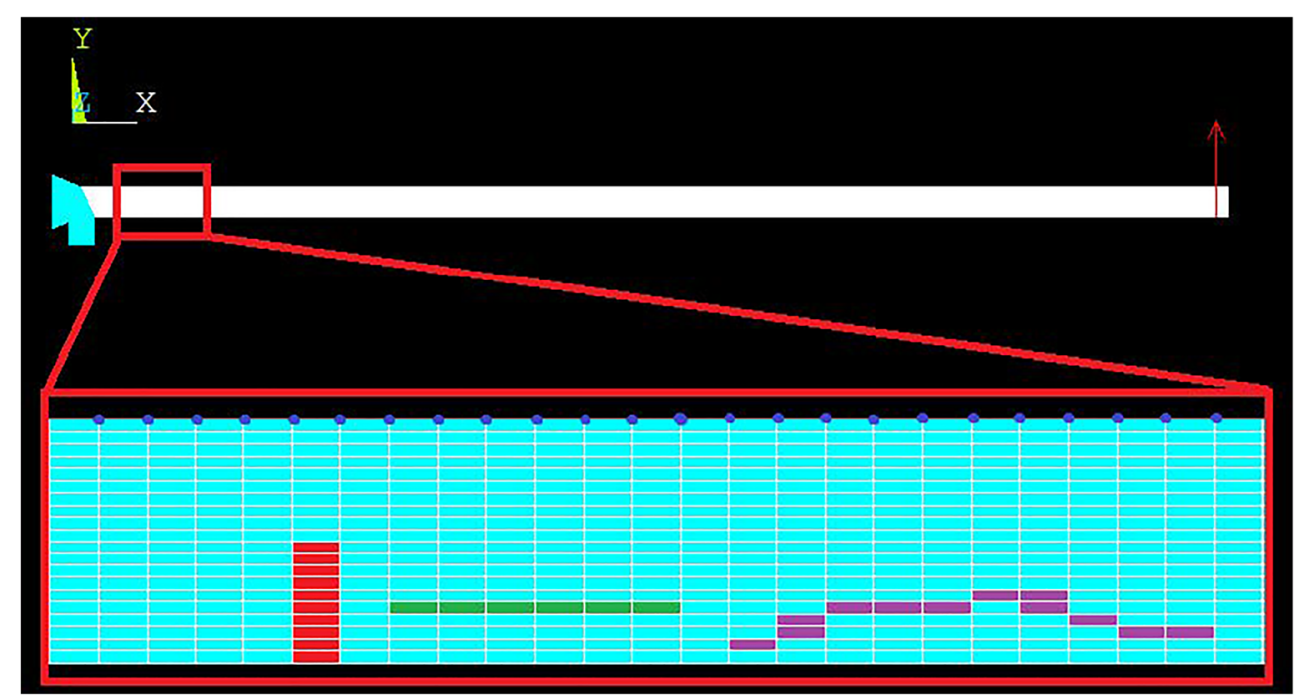


Table 4 Frequency and Phase variation depending on the damage severity

\begin{tabular}{llllllll}
\hline Eigen & \multicolumn{2}{l}{ Frequency $[\mathrm{Hz}]$} & & & \multicolumn{2}{l}{ Phase [deg] } \\
\cline { 2 - 3 } \cline { 7 - 8 } \cline { 6 - 8 } & D10T & D25T & D50T & & D10T & D25T & D50T \\
\hline$\omega_{1}$ & $0.0 \%$ & $2.0 \%$ & $2.0 \%$ & & $41.0 \%$ & $51.8 \%$ & $53.5 \%$ \\
$\omega_{2}$ & $0.3 \%$ & $0.3 \%$ & $0.6 \%$ & & $19.7 \%$ & $35.2 \%$ & $40.5 \%$ \\
$\omega_{3}$ & $0.0 \%$ & $0.1 \%$ & $0.2 \%$ & & $5.2 \%$ & $14.0 \%$ & $21.4 \%$ \\
$\omega_{4}$ & $0.0 \%$ & $0.0 \%$ & $0.0 \%$ & & $-2.1 \%$ & $-4.3 \%$ & $-5.5 \%$ \\
$\omega_{5}$ & $0.0 \%$ & $0.1 \%$ & $0.1 \%$ & & $3.1 \%$ & $10.5 \%$ & $18.3 \%$ \\
$\omega_{6}$ & $0.1 \%$ & $0.2 \%$ & $0.4 \%$ & & $11.1 \%$ & $26.7 \%$ & $32.4 \%$ \\
$\omega_{7}$ & $0.2 \%$ & $0.3 \%$ & $0.6 \%$ & & $16.7 \%$ & $32.4 \%$ & $36.3 \%$ \\
$\omega_{8}$ & $0.2 \%$ & $0.4 \%$ & $0.7 \%$ & & $18.1 \%$ & $32.5 \%$ & $36.2 \%$ \\
\hline
\end{tabular}

beam excited at its tip the shape is such to have a constrained and hinged endpoints respectively, as shown in Fig. 10. Despite very low deflection amplitude for the shape at the anti-resonance, one can observe curvature in the proximity were the damaged was positioned. Therefore, following this approach, the strain energy analysis was carried out on all resonances and anti-resonances.

The resonance and anti-resonance frequencies are compared to each other, and a table of the strain energy at the damage location is made, see Table 5. The strain energy analyses are conducted for the pristine condition since the damage model would inevitably show a spike of strain energy at the damage location. The values at the damage location are listed for all resonances and anti-resonances. As it appears from Table 5, the strain energy calculated at the anti-resonances would be higher than the one calculated for the resonances for high frequency ranges. The fifth column of Table 5 portrays the percentage increase or decrease (-) of strain energy between the resonance and the following anti-resonance. The sixth column shows this difference between the antiresonance and its following resonance. By close inspection, one could notice that the strain energy at the anti-resonances is typically higher than the ones at the resonances from mode four and onwards.

The differences highlighted in Table 5 become evident when the ODSs at resonant and anti-resonant conditions and strain energies are plotted. Figure 11 shows the ODSs for the fifth, sixth resonances and at the anti-resonance in between them, respectively and, as expected, the ODS magnitude of the anti-resonance is negligible. The strain energy plot, see Fig. 12, shows that its overall magnitude calculated at the anti-resonance frequency is higher than the ones at resonance. This investigation will aid the understanding of the next sections where the CSLDV will be used in frequency ranges spanning from resonances and anti-resonances. As we know, the amplitude modulated LDV output signal will give spectral sidebands at any excitation frequency. It is assumed that the sidebands relative magnitudes (let us say with respect to the centre one) can indicate changes of curvature between a pristine and damaged case. Hence, the next section will discuss how to exploit the spectral sidebands for detecting the presence of damage directly.

\section{Development of RASTAR Method}

This section will deal with the development of the Relative Amplitude of the Sidebands to the Total Amplitude Reference (RASTAR) method. It is based on the analysis of spectral sidebands obtained by CSLDV measurements performed to pristine (or know condition) and damaged cases. A damaged condition does not necessarily mean that the structure is unsafe or unable to meet the structural performances; it just defines a state of changing. The goal is to exploit the information of the spectral sidebands without going through polynomial transformation for ODS recovery. The attempt focuses on a straight-line scan method, but it can be extended to twodimensional scans.
Fig. 3 Response function for pristine and damaged cases, Amplitude and Response phase
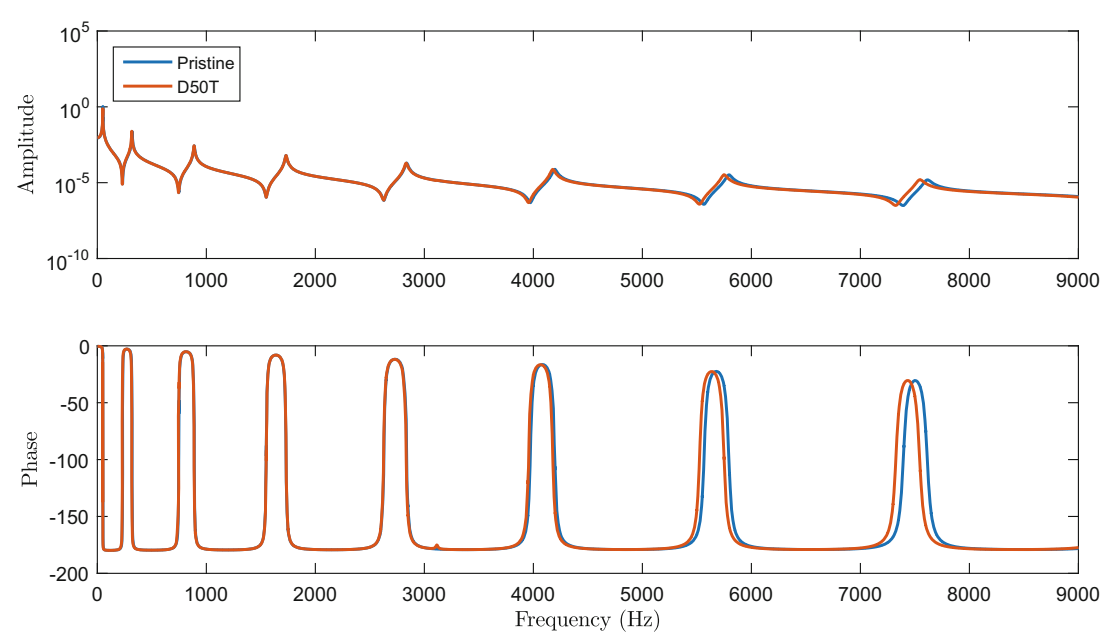


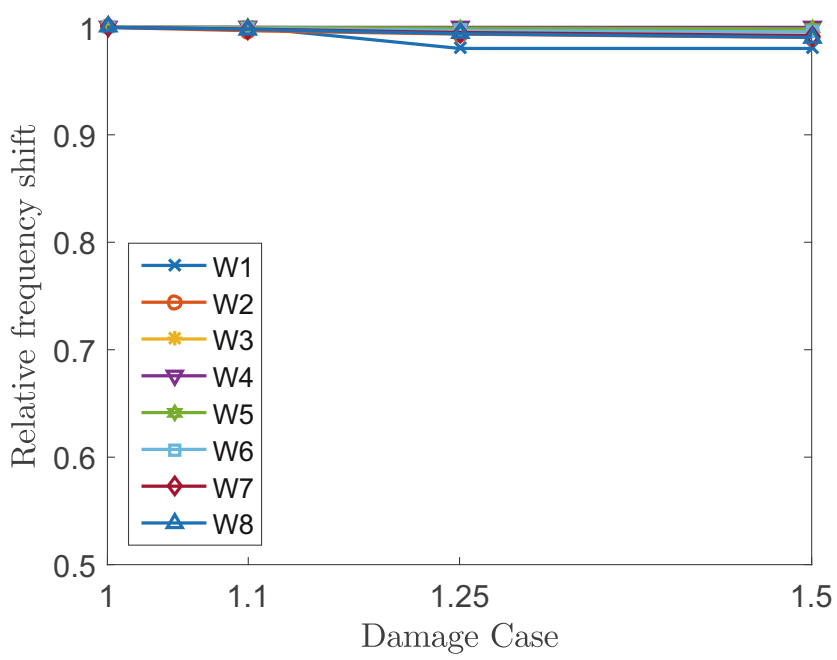

Fig. 4 Relative frequency shift

\section{Simulation of Continuous Scanning LDV Output Signal}

The next phase in simulating the output spectrum is extracting the Operational Deflection Shapes (ODSs) from the FE model, and this is done by performing a harmonic analysis and evaluating the $y$-displacement of all the top row nodes for all frequencies covering up to the 8 th resonance. The $x$ coordinate of the beam is normalised between -1 at the root and 1 at the tip. The normalisation makes 0 the middle of the beam and normalises the beam length to 2, using Eq. (1). The operation is done to allow for simulation of the continuous sinusoidal line-scan.

$x_{\text {norm }}=$ Scale $\cdot(x)+$ Offset

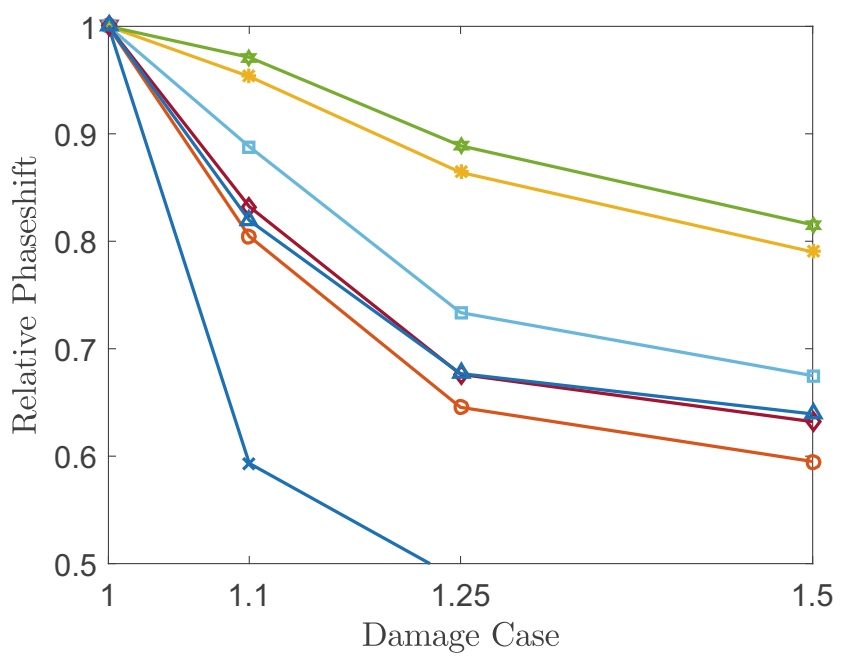

Fig. 5 Relative response phase shift
Where:

Scale $=\left(\frac{1}{\frac{1}{2}(\operatorname{Max}(x)-\operatorname{Min}(x))}\right) ;$ Offset $=-1$

The normalised length is used as the new $x$-coordinate of the ODS. Now a polyfit -function is used in Matlab to obtain the polynomial coefficients of the ODS shape. This function approximates the shape with a polynomial, like in Eq. (3).

$p(x)=A_{0}+A_{1} x+A_{2} x^{2}+\ldots$

The number of polynomial coefficients needed to describe the ODS adequately is dependent on the frequency since a higher frequency vibration shape is more complex and thus requires more polynomial coefficients. The minimum number needed is calculated by the Modal Assurance Criterion (MAC), Eq. (4), which relates the approximate shape to the real shape [30], the MAC value necessary to describe the eigenfrequencies is evaluated to get an idea of the necessary number of coefficients. The number of coefficients is increased iteratively as long as the MAC value is below 0.99 .

$M A C=\frac{\left(\Phi_{a}^{\mathrm{T}} \cdot \Phi_{r}\right)^{2}}{\Phi_{a}^{\mathrm{T}} \cdot \Phi_{a} \cdot \Phi_{r}^{\mathrm{T}} \cdot \Phi_{r}}$

Where $\Phi_{a}$ is the ODS approximated by a polynomial and $\Phi_{r}$ is the ODS obtained from the FE model. The results are shown in Table 6, where the minimum number of polynomial coefficients are listed for every resonance. It was decided to simulate the CSLDV line scan with a fifteen coefficient to assure all ODSs from the model can be correctly fitted. Clearly, modes in low frequency ranges will present LDV modulations with several spectral lines in the floor noise (either numerical or superimposed noise) which will be of no use, but these extra sidebands will not be detrimental to the analysis.

The deflection shapes simulated at 9000 frequencies $(1 \mathrm{~Hz}$ frequency spacing) are approximated by polynomial functions, the amplitude modulated time signal can be generated for a continuously sinusoidal line-scan. The CSLDV measures the velocity of the vibration, and this can be written as Eq. (5).

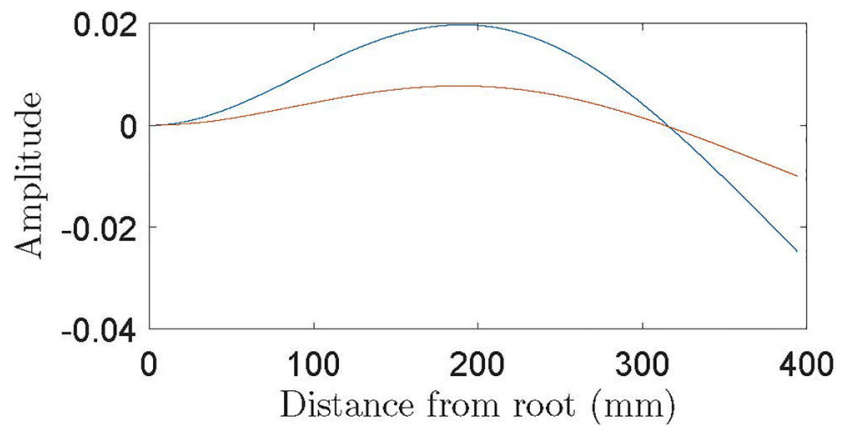

Fig. 6 ODS of the 2nd bending mode. Pristine(blue) damaged (red) 


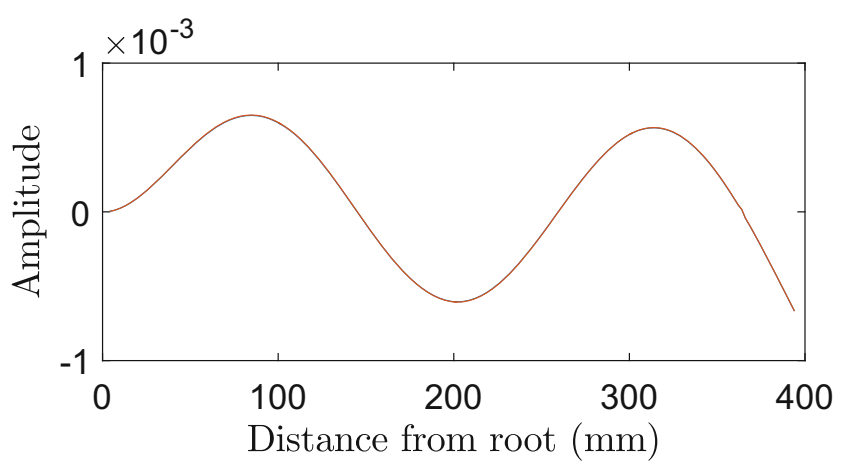

Fig. 7 ODS of the 4th bending mode. Pristine(blue) damaged (red)

$v(t)=\sum_{p=1}^{N} A_{p} \cos ^{p}\left(\Omega_{\mathrm{x}} t\right) \cos (\omega t)$

Where $\Omega_{\mathrm{x}}$ is the scan rate along the $\mathrm{X}$-direction, $N$ is the number of coefficients (set to 15) and $A_{p}$ is the polynomial coefficient. Since the amplitude of the sidebands carries this information, the distribution of these sideband amplitudes can be considered characteristic for a specific system at a fixed excitation frequency $[14,31]$. When damage is introduced to the structure, its dynamic response changes, and thus, the spectrum changes [24]. This can be seen in Fig. 13. The blue stems show the pristine spectrum and the red ones show damaged case; both spectra were excited at the pristine second eigenfrequency.

The spectral lines representing the damaged case are lower than the pristine ones. This behaviour is expected because the amplitude of the FRF at the pristine eigenfrequency is higher than for the damaged instance, as seen in Fig. 14, and remembering that is the same excitation frequency for both cases.

This can be described as an "Eulerian" approach where one would observe changes of structural integrity from a fixed reference point. Hence, when the response is measured at the pristine eigenfrequency, the excitation amplitude is no longer at a peak if the beam is damaged, explaining the lower overall magnitude of the damaged sidebands. More importantly, it is that the distribution profile of the sideband amplitudes has clearly changed from its characteristic pristine case, see Fig. 13 from the sixth sideband to the 15 th one. The insight that the spectrum changes from its characteristic shape when damage is introduced to the system can be exploited for SHM purposes. The next step is developing a damage indicator that computes this change in sideband amplitude distribution.

\section{RASTAR Method}

Several attempts were made prior to the development of RASTAR, but these are already reported in [32]. This method was developed after the fingerprint method in [32], and RASTAR stands for Relative Amplitude of the Sidebands to the Total Amplitude Reference. Where the fingerprint method based its normalisation on only one (the middle) sideband, this method makes use of all sidebands. To normalise the amplitudes of both cases, first, the amplitude of all sidebands in the spectrum is summed, referred to as the total amplitude. The individual sideband amplitude is calculated as a percentage of the total amplitude; every sideband is assigned a percentage value of its relative contribution to the total amplitude. This is expressed in Eq. (6). By doing so for the pristine and damage cases, the relative contribution percentages per sideband can be compared. The damage indicator for that frequency is acquired by summing all differences per relative sideband. A lesson learned from the polynomial sideband method is that this RASTAR method can be applied for any number of sidebands chosen. As said before, at lower frequencies, most information is in the first few sidebands, so only using the method for these sidebands would not change the sensitivity much. It would change for higher frequencies since more sidebands are needed to describe the shape.
Fig. 8 Strain Energy of the 2nd bending mode- pristine

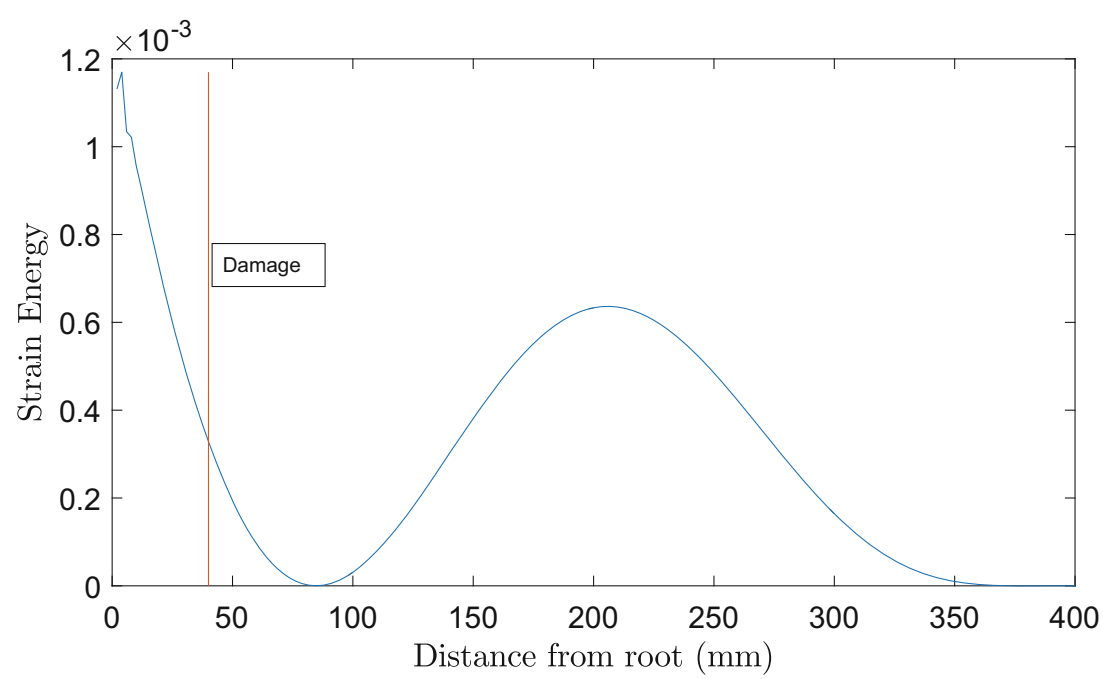


Fig. 9 Strain Energy of the 4th bending mode- pristine

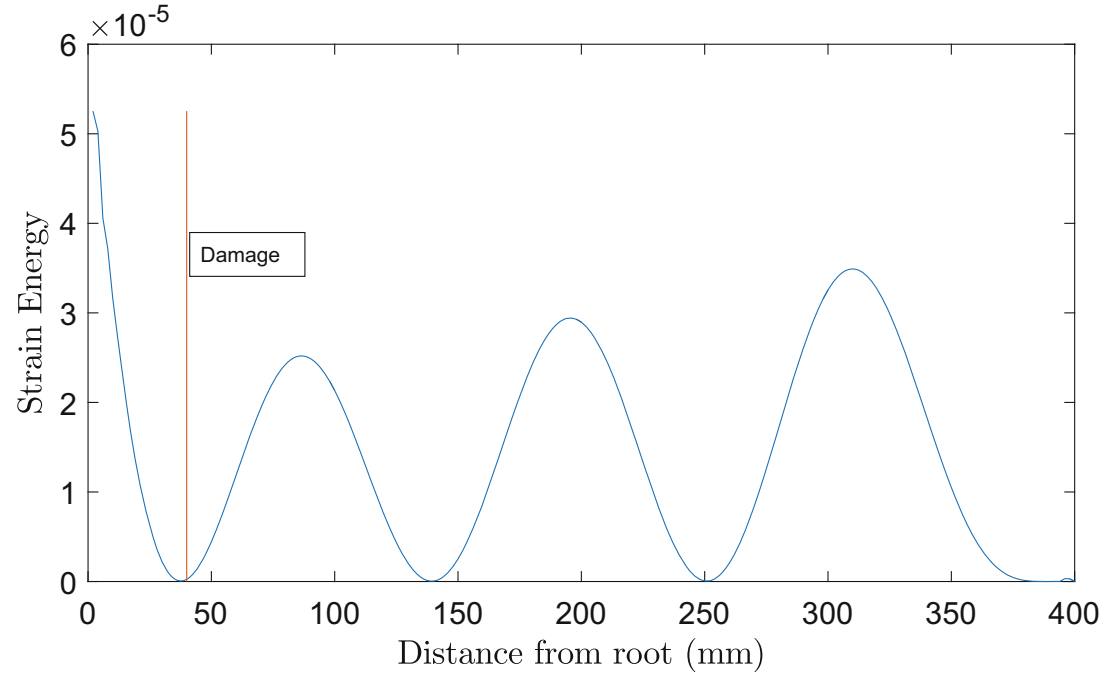

$\epsilon(t)=\sum_{j=1}^{n}\left(\frac{S B A_{P}(\omega \pm \Omega j)}{\sum_{i=1}^{n} S B A_{P}(\omega \pm \Omega i)} \cdot 100 \%-\frac{S B A_{D}(\omega \pm \Omega j)}{\sum_{k=1}^{n} S B A_{D}(\omega \pm \Omega k)} \cdot 100 \%\right)$

Where the subscript $P$ is Pristine, $D$ is Damaged, and $n$ is the number of sidebands.

The RASTAR algorithm exploits that physics of the spectral sidebands which describe the spatial distribution of the deflection (and the deformation) shapes as it changes because of an occurring structural change.

\section{Application of RASTAR to Simulated Spectral Sidebands}

As briefly introduced earlier, the simulations were conducted in a frequency range between 1 and $9000 \mathrm{~Hz}$, with a frequency step of $1 \mathrm{~Hz}$, with an excitation force applied to the tip of the beam and an excitation amplitude of $1 \mathrm{~N}$. The ODS simulated by the harmonic analysis were fitted with a polynomial of the 15 th order and the polynomial coefficients used for simulation the modulated LDV output signal. The spectral sidebands were fed to the RASTAR algorithm, and plots of Summed Difference vs Frequency were created.

The performance of RASTAR was evaluated between the Pristine and the Damaged cases with severities of 10\%, 25\% and 50\%, and the results are shown in Fig. 15. As expected, the increasing damage severity leads to an increase of the damage indicator. Furthermore, the peaks that are noticeable in Fig. 15 are in the anti-resonance regions, that as indicated earlier are regions very sensitive to the presence of damage for the case study presented in this paper. The halving of the excitation force did not change the performance of the damage indicator but just its magnitude, where.

Figure 16 shows the indicator calculated for pristine to pristine and pristine to damaged $10 \%$. As can be seen, the blue line, which represents the damage indicator for the pristine case compared to the pristine case at low force shows null values. Actually, we considered that just changing the excitation force could change the relative distribution of the amplitudes of the sidebands. By taking the pristine case at $1.0 \mathrm{~F}$ and the pristine case at $0.5 \mathrm{~F}$ and using RASTAR on it (blue line) one can see that there is no damage indication due to the change in excitation force in comparison to the standard pristine $1.0 \mathrm{~F}$ vs. D10T $1.0 \mathrm{~F}$ case. So the blue line is pristine vs pristine at a different excitation level to show the independence of the method in terms of excitation force. The RASTAR is insensitive to the vibration amplitude, as explained before, the relative magnitudes of the sidebands bear the insight of the damage, that leads to the question on how sensitive is RASTAR to the number of sidebands. For this reason, RASTAR was also evaluated for different numbers
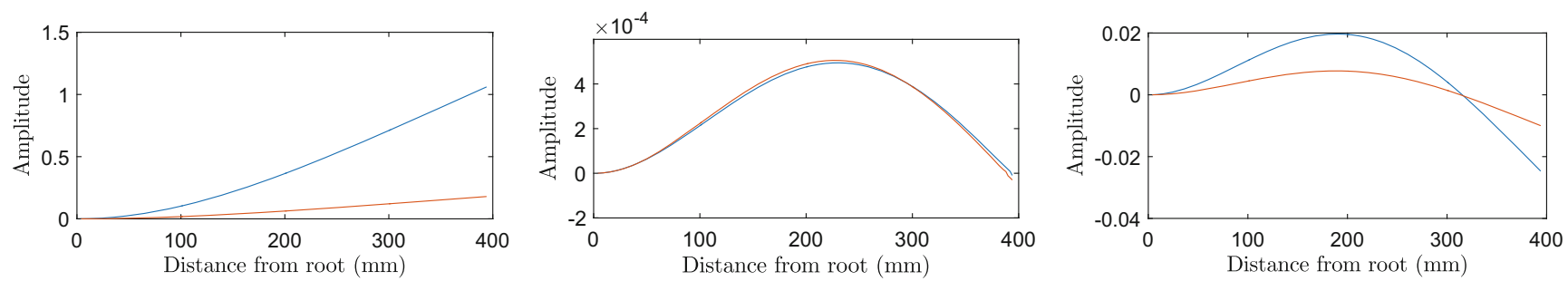

Fig. 10 First bending (a), bending at the anti-resonance (b) and second bending (c) 
Table 5 Strain Energy differences between resonance and antiresonance

\begin{tabular}{clllrl}
\hline Res. (n) & $\begin{array}{l}\text { Strain } \\
\text { Energy } \\
\left(10^{-6}\right)\end{array}$ & Anti Res. (n) & $\begin{array}{l}\text { Strain } \\
\text { Energy }\left(10^{-6}\right)\end{array}$ & $\Delta$ SE n:n & $\begin{array}{l}\Delta \mathrm{SE} \\
\mathrm{n}+1: \mathrm{n}\end{array}$ \\
\hline 1 & 448,540 & 1 & 49.6970 & $-99.98 \%$ & $-87.87 \%$ \\
2 & 409.62 & 2 & 3.9423 & $-99.04 \%$ & $236.92 \%$ \\
3 & 1.1701 & 3 & 0.0095 & $-99.18 \%$ & $-96.16 \%$ \\
4 & 0.2477 & 4 & 0.8234 & $232.42 \%$ & $213.08 \%$ \\
5 & 0.2630 & 5 & 2.1810 & $729.28 \%$ & $171.37 \%$ \\
6 & 0.8037 & 6 & 3.1822 & $295.94 \%$ & $61.44 \%$ \\
7 & 1.9711 & 7 & 3.5857 & $81.91 \%$ & $28.79 \%$ \\
8 & 2.7842 & & & & \\
\hline
\end{tabular}

of sidebands used for the total summation. The effect of changing the number of sidebands included in the total summation can be seen in

Figure 17, and all three are the pristine case compared to the D50T damage case. As expected, the lower frequencies show little change from using more sidebands, since the higher-order terms do not hold much meaning for these frequencies. However, even for the higher frequencies, the lower amount of sidebands taken into account seems to result in a more sensitive indicator at the anti-resonances. RASTAR was also investigated when white noise was added to the simulated LDV signal as to be more representative to a real-life condition. It is worth reminding that white background noise affects the amplitudes of the sidebands randomly, and it is necessary to check how sensitive RASTAR is to that condition. Figure 18 shows the performance evaluated over the $9 \mathrm{kHz}$ frequency range, where one can see that at the lower damage severity the indicator falls in the same range of values of the pristine case, evaluate against itself, for higher frequency range. However, not at the resonances as signal-to-noise ratio is better due to the higher amplitudes. For frequency range, up to $1 \mathrm{kHz}$, the performance is a little better for the $10 \%$ damaged case as Fig. 19 shows.

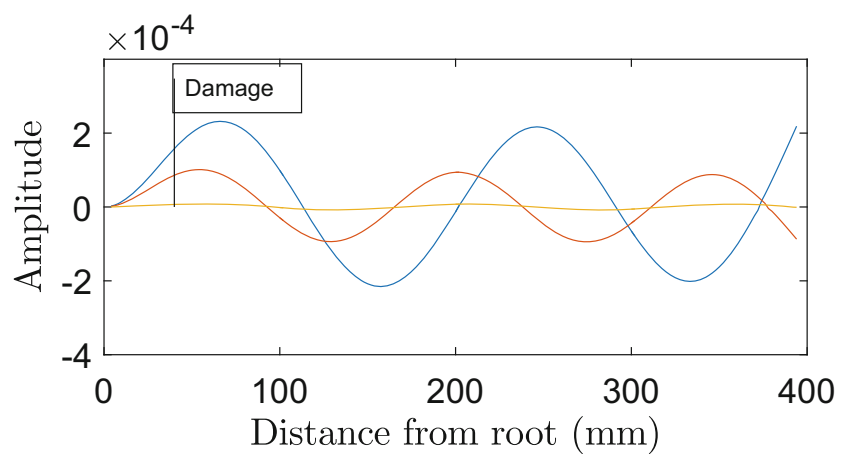

Fig. 11 ODSs of the 5th (blue) and 6th (red) resonances and the ODS of the anti-resonance (yellow)

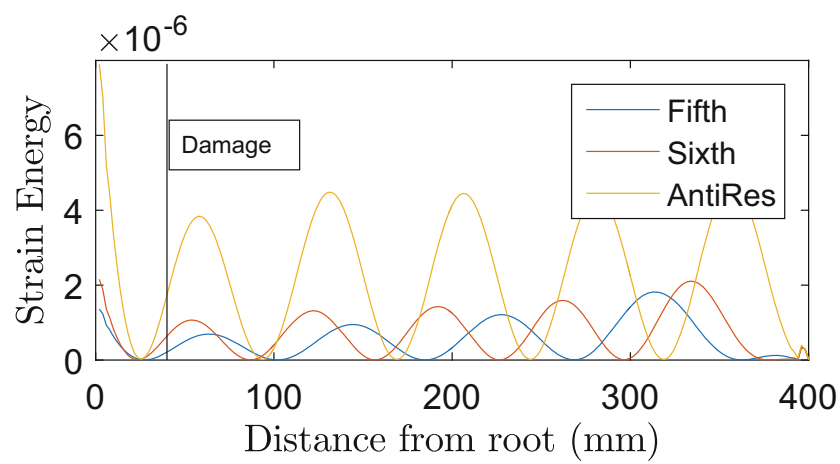

Fig. 12 Strain Energy of the ODS at resonance and anti-resonance

The last case reported in this section is the performance of RASTAR when a longitudinal damage case is investigated, bearing in mind that this is a pure artefact since an aluminium beam would not develop such damage. The damage was not elongated but deepened from the $10 \%$ up to $50 \%$ in the width direction, as showed in Fig. 2. Figure 20 shows the result of the simulation, and the performance of RASTAR is similar to the previous cases analysed. Still, in here, the magnitude of the damage index is lower than for the transverse damage case.

\section{Experimental Verification of the RASTAR Method}

The results obtained in the numerical study are verified by experimental analysis. The experiments are conducted with the knowledge that the result will differ from the numerical model, but the general behaviour is expected to be the same. For instance, the peaks of the indicator at the anti-resonances as found in the numerical analysis are not likely to be as high, mainly because of the lower expected signal-to-noise ratio.

\section{Test Set-up and Experimental Parameters}

For the experimental analysis, a test set-up was built. A steel L-shape frame was available in which the samples could be clamped. The frame was bolted to a metal plate using steel framework and bolts to fasten it down. The metal plate is fixed to a concrete block on rubber legs to damp most vibrations from the room. The specimen is an aluminium beam of $30 \mathrm{~mm}$ wide and $10 \mathrm{~mm}$ thick. The length is approximately $500 \mathrm{~mm}$, but the beam is clamped to have a sufficient length of $400 \mathrm{~mm}$. The difference in width to the simulation does not matter for the eigenfrequencies, as explained with Eq. (7).

Table 6 Minimum number of polynomial coefficients

\begin{tabular}{lllllllll}
\hline Eigen-Frequencies & 1 & 2 & 3 & 4 & 5 & 6 & 7 & 8 \\
Nr. of coefficients & 2 & 4 & 5 & 8 & 9 & 10 & 13 & 14
\end{tabular}


Fig. 13 Spectral sidebands of the pristine and damaged case $(50 \%)$

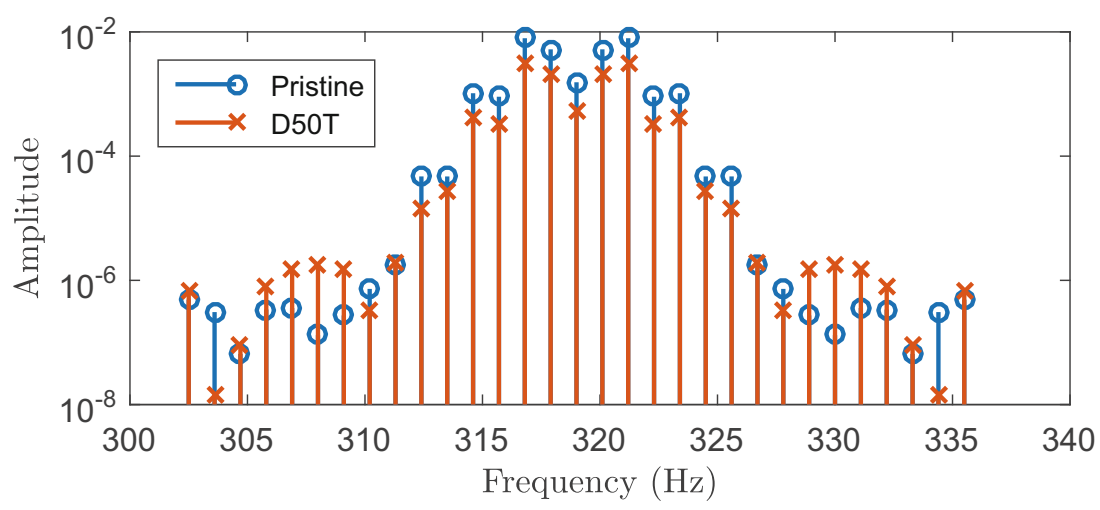

$\omega_{i}=A_{i}^{2} \sqrt{\frac{E \frac{h^{3}}{12}}{h l^{4} \rho}}$

The beam is categorised as aluminium 6082-T6. The laser used for the experiment is the Polytec PSV-I-560. The laser is positioned sufficiently far from the rig, so the mirror rotation allows for the laser to scan the entire length of the beam. Both the laser path and the shaker excitation point are placed along the torsional line to minimise as much as possible any torsional vibrations. The shaker excitation is at the tip of the beam which connects with the force gauge. A picture of the experimental set-up is shown in Fig. 21.

The experiments are conducted for six cases, three damage severities and two scan rates. The damage cases are: Pristine, a $1 \mathrm{~mm}$ by $1 \mathrm{~mm}$ cut at $40 \mathrm{~mm}$ from the root, and a $2 \mathrm{~mm}$ by $2.5 \mathrm{~mm}$ cut at $40 \mathrm{~mm}$ from the root. The scan rates are set at $1.1 \mathrm{~Hz}$ and $0.9 \mathrm{~Hz}$. All tests are carried out using the same beam, which was removed when the damage was machined by milling. Conducting the experiments for the full frequency range used in the numerical analysis is not considered viable. Aluminium does not have a fatigue limit, and fatigue will start to be a factor if the full range would be tested for multiple damage severities. Considering this and limited time, a frequency range is defined. The tests are done from 20 to $1000 \mathrm{~Hz}$ with a frequency step of $1 \mathrm{~Hz}$. In this frequency range, the numerical results indicate a clear difference between the damage severities, even in the presence of noise, because the excitation amplitude of the beam is relatively high in this region, yielding a favourable signal-to-noise ratio. The trade-off is that in the simulation, the higher frequencies result in the highest peaks in the damage indicator. In the region below $1000 \mathrm{~Hz}$, the settings of the laser precision, sample rate and force amplification can be kept constant and still yield good results.

Several FRFs (see Fig. 22) are measured with the SLDV in a frequency range of $0-2000 \mathrm{~Hz}$. These data are processed to identify both the eigenfrequencies and the anti-resonances. The FRF shows more peaks because of torsional modes and some test rig modes. Then, the test is done for the specified frequency range, and the time signals are saved for every frequency. Every frequency is scanned for $10 \mathrm{~s}$ with a sample time of $1 \times 10^{-4} \mathrm{~s}$. After completing the tests using both scan rates, the beam is removed from the rig and milled to set damages as mentioned. The time signal is post-processed in the same manner as in the simulation, a dFFT at the specific scan rate up to 15 sidebands is performed. To reduce noise, the left- and its respective right-hand sidebands are averaged, since these should be equal. The damage indicator is calculated for all frequencies; the results are discussed in the following section.
Fig. 14 Frequency response of pristine and damaged case
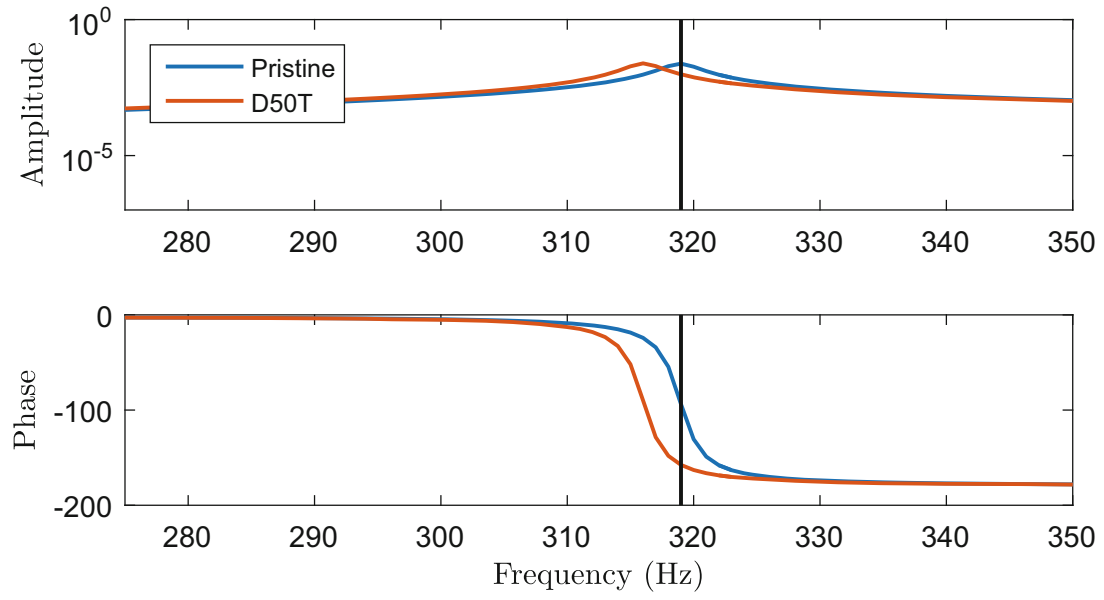
Fig. 15 RASTAR results for different damage severities

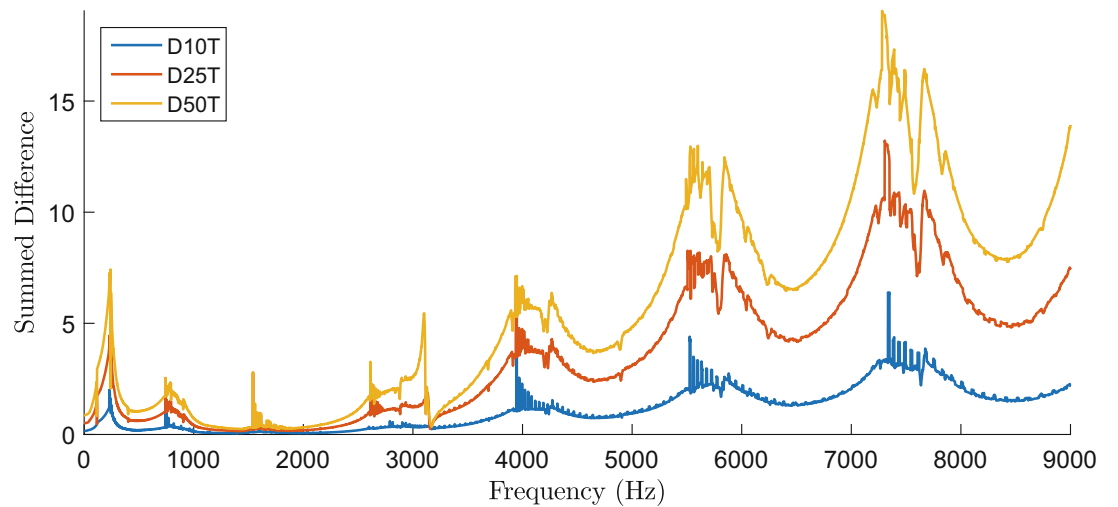

\section{Experimental Results}

First, for the benchmark case, the pristine case of $1.1 \mathrm{~Hz}$ scan rate is compared to the $0.9 \mathrm{~Hz}$ scan rate. The result shown in Fig. 23 behaves as expected, at the resonances of the beam, the signal-to-noise ratio is very high, resulting in a low damage indicator. In between the resonances, a higher indicator value is reached due to the noise.

The output spectra of the pristine case at the $1.1 \mathrm{~Hz}$ scan rate are compared to the $1 \mathrm{~mm}$ damage case and the $2.5 \mathrm{~mm}$ damage case. This is done in the same manner as described above in the indicator definition using Eq. (6) for the RASTAR method, and the first eight sidebands are included in the calculation. The result is viewed below in Fig. 24, where the blue line represents the benchmark case. The red line shows the damage indicator of the $1 \mathrm{~mm}$ damage case to the pristine case and the yellow line the $2.5 \mathrm{~mm}$ damage case to the pristine case. In the ideal situation, the yellow line should yield the highest value on the damage indicator, the red line the second-highest and the blue line the lowest over the entire frequency range, this is the case for most frequencies.

However, one observation that immediately stands out from the results is that many peak values appear very periodically. The spikes in the experimental results of the damage cases are all linear combinations of $11 \mathrm{~Hz}$, or a tenfold of the scan rate of $1.1 \mathrm{~Hz}$. This can be explained as the scan rate harmonic speckle noise. Martarelli et al. published on speckle noise related to the scan rate in 2006 [33]. It was stated that noise is expected in the LDV output signal at the scan rate and its multiple harmonics. Therefore, periodic noise is introduced by the scan rate of $1.1 \mathrm{~Hz}$, and at its multiple harmonics. Because of the discrete sideband operations in the damage indicator, the scan rate harmonics coincide precisely with the sidebands at that harmonic excitation frequency. The noise at exactly these frequencies contribute to a changing spectrum shape on which the damage indicator is based, resulting in a higher damage indicator value. The trend of the damage indicator result would be more clear without this speckle noise. Therefore these peaks should be filtered out. The periodicity of these peaks can be more clearly seen when an FFT of the damage indicator result itself is taken over the frequency range. The result for the $1 \mathrm{~mm}$ damage case is viewed in Fig. 25. The peaks can immediately be distinguished; however, the $\mathrm{x}$-axis needs to be modified to present the correct frequencies. As implemented above, an FFT takes a time signal and transforms it into a frequency spectrum of that signal. In this case, the input is in the frequency domain, so the output should be modified. The input is treated as a time signal of $1 \mathrm{~s}$, and the measurements range from $20 \mathrm{~Hz}$ to $1000 \mathrm{~Hz}$, this means 981 sample point. So a 'sampling frequency' of
Fig. 16 Damage indicator halving the excitation force

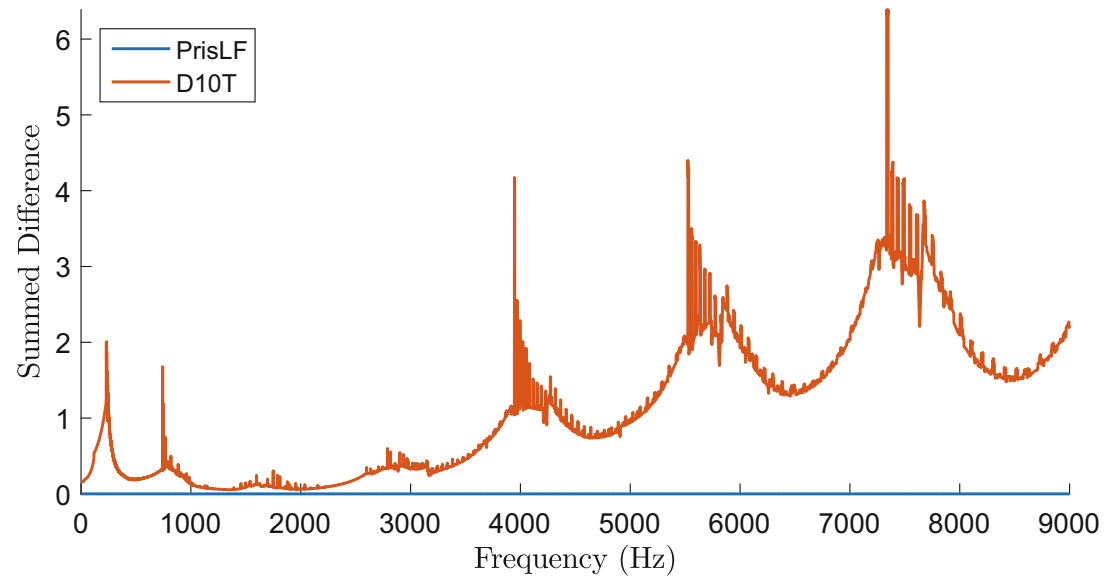


Fig. 17 Damage indicator performance for different numbers of summed sidebands

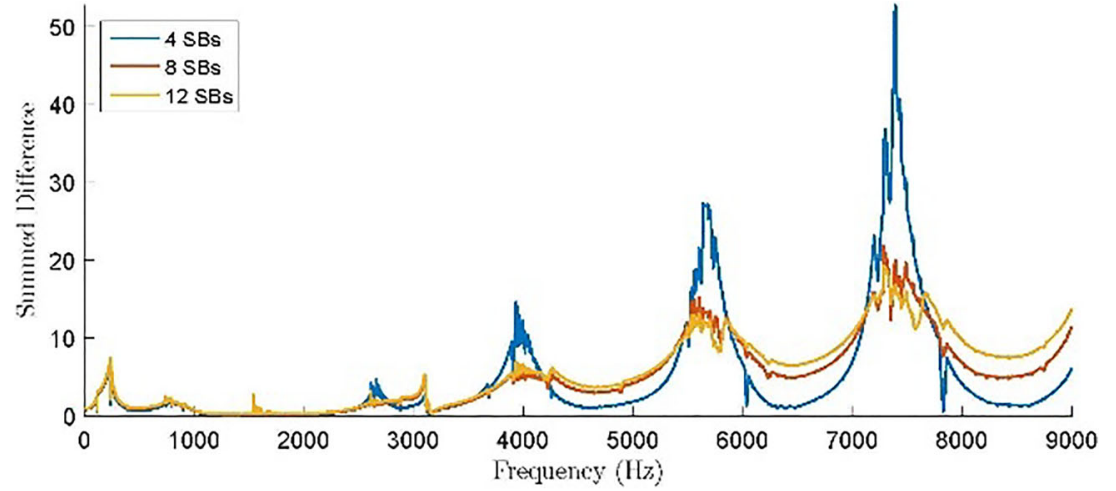

$981 \mathrm{~Hz}$. An FFT of such a signal results cuts off at a Nyquist frequency of half that, $491 \mathrm{~Hz}$. The first peak appears at $90 \mathrm{~Hz}$ in the unmodified FFT. This means a period of $1 \mathrm{~s}$. This multiplied with the number of samples: 981 , equals nearly $11 \mathrm{~Hz}$. When the $\mathrm{x}$-axis is modified to represent that, the peaks at the $11 \mathrm{~Hz}$ harmonic become clear, as seen in Fig. 26. The graph is now also cut off at $491 \mathrm{~Hz}$, the expected Nyquist frequency.

Notch filters were placed at these harmonics to make the graph smoother. This filtering process will make the behaviour of the damage indicator more clear. The filtered FFT is also plotted in Fig. 26, which gives a better sense of the general, underlying behaviour of the damage indicator. These filters are also applied to the benchmark case. An important thing to note is that the comparison of the pristine case consists of a scan at $1.1 \mathrm{~Hz}$ and a scan at $0.9 \mathrm{~Hz}$, meaning that the speckle noise harmonics at $9 \mathrm{~Hz}$ should also be filtered as well. The result of the filtered RASTAR data for LDV scan rate of $1.1 \mathrm{~Hz}$ and $0.9 \mathrm{~Hz}$, respectively, are shown in Figs. 27 and 28.

\section{Discussions}

This section will focus on the discussion of the experimental results. Figures 27 and 28 show the RASTAR data obtained from tests executed at two different LDV scan rates, $1.1 \mathrm{~Hz}$ and $0.9 \mathrm{~Hz}$, respectively. In both cases, the RASTAR indicator shows the difference between pristine and damaged cases. The overall amplitude of the indicator tends to increase for high frequency ranges, showing very distinctive peaks in the $700 \mathrm{~Hz}$ frequency range, which are also present in Fig. 28, where the scan rate was changed. By looking at both figures, Figs. 27 and 28, the sensitivity to the damage severity would be expected to be higher for larger damage, but it is not the case for the entire frequency range tested when either $1.1 \mathrm{~Hz}$ or $0.9 \mathrm{~Hz}$ scan rates are attempted. In some frequency ranges, RASTAR analysis seems to be more sensitive for smaller damages than larger ones. As overall, RASTAR is capable of detecting the change in structural integrity, but further investigation will be required to underpin the physics creating such differences.

A comparison between the RASTAR indicator and the FRF (pristine conditions) is performed and showed in Fig. 29, where the $1 \mathrm{~mm}$ damage case is plotted over the FRF up to $1000 \mathrm{~Hz}$. There are a few frequencies that stand out. These are at the peaks in the indicator at $267 \mathrm{~Hz}$ and $678 \mathrm{~Hz}$, where the damage indicator is close to the antiresonances at $225 \mathrm{~Hz}$ and $717 \mathrm{~Hz}$. First, the experimental FRF and its resonances do not align well with the analytically calculated eigenfrequencies, shown in Table 7. Those
Fig. 18 RASTAR index for noisy LDV signals

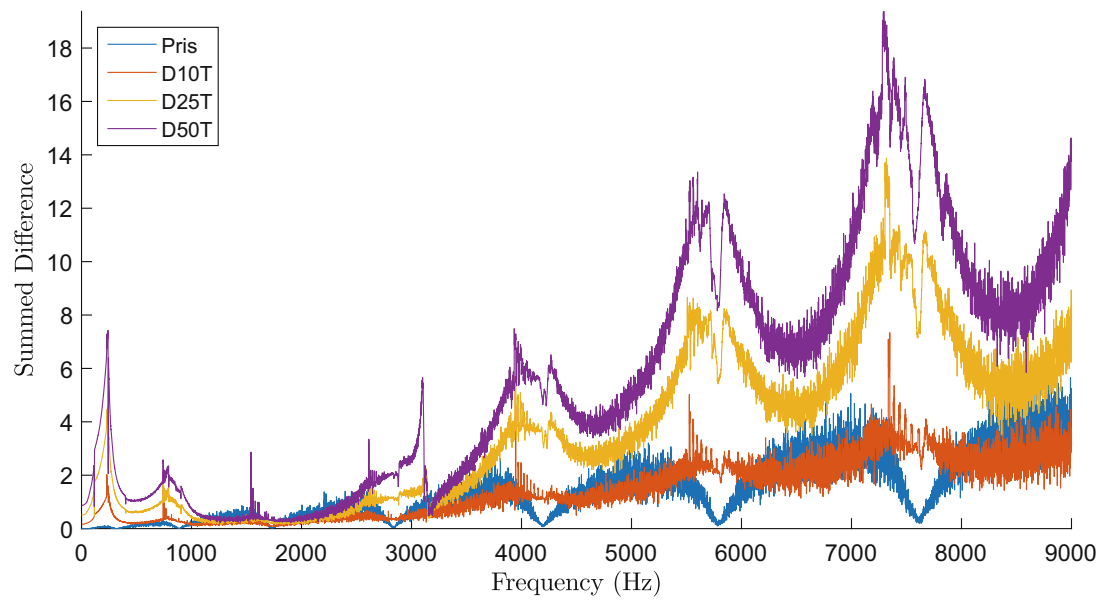


Fig. 19 Zoom of Fig. 18

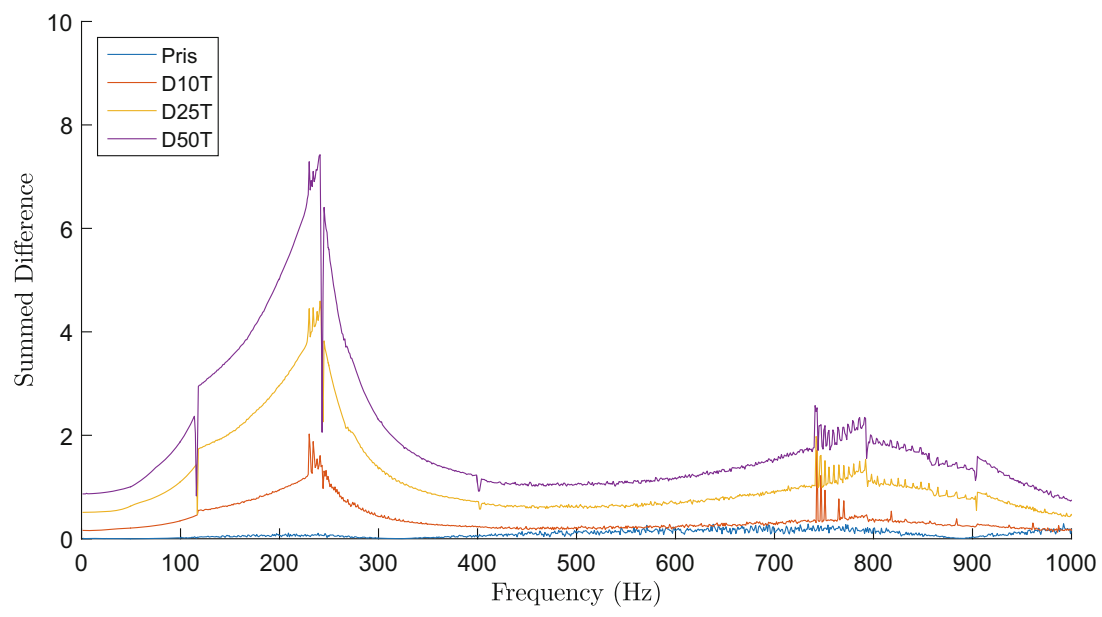

differences were expected because of (i) no model updating (ii) test rig compliance and (iii) beam clamping conditions which slightly changed when the damages had to be made by a milling machine.

By observing the plot in Fig. 29, the low noise between 700 and $800 \mathrm{~Hz}$ for the pristine case should indicate the presence of a resonance, namely high signal-to-noise ratio due to the large excitation amplitude. This resonance does not indeed line up with the peak of the third eigenfrequency in the FRF as it does in the numerical analysis. It also seems to be the case for the second eigenfrequency. If the FRF were to shift left to accommodate for this, the peak in the indicator does line-up with the second anti-resonance, but the first anti-resonance would not line-up with the peak at $267 \mathrm{~Hz}$ in the D10T damage case. These observations do not undermine the performances of RASTAR as a damage indicator, as the profile of the $10 \%$ damage conditions stands above the pristine one. However, further investigations will be carried out to evaluate such mismatches.

The general behaviour of the pristine case, compared to another pristine case correlates very well to the numerical results with added random noise. The noise at frequencies between the resonances results in a relatively high damage indicator. The signal-to-noise ratio increases and the damage indicator becomes lower at and around the eigenfrequencies. Such behaviour was also shown in the numerical results; the overall damage index profile is similar, as displayed by the blue line in Fig. 18.

Another difference is found in the overall shape between the damage indicator results of the experimental and numerical analyses. This is because of a fundamental difference in the way the experiments are conducted, and the simulation is performed. In the simulation, a set force magnitude is applied to all frequencies, while in the experiment, the actual force magnitude differs for every frequency. This is part of the reason why the indicator had to include some sort of normalisation to the force input level, which would make RASTAR less sensitive to that parameter. This is also why the signal to noise ratio is so high for the first $1000 \mathrm{~Hz}$ in the model.

A final remark is about the LDV output noise from the continuous scanning acquisitions. Two types of noises were clearly visible in all plots presented, one was caused by the scan rate, which could be filtered out, and the other one is inherently present in the sidebands spectra which RASTAR processing seems enhancing. The noise in the form of spikes that are the results of the RASTAR analysis can be somehow
Fig. 20 RASTAR evaluated for a delamination lookalike type of damage

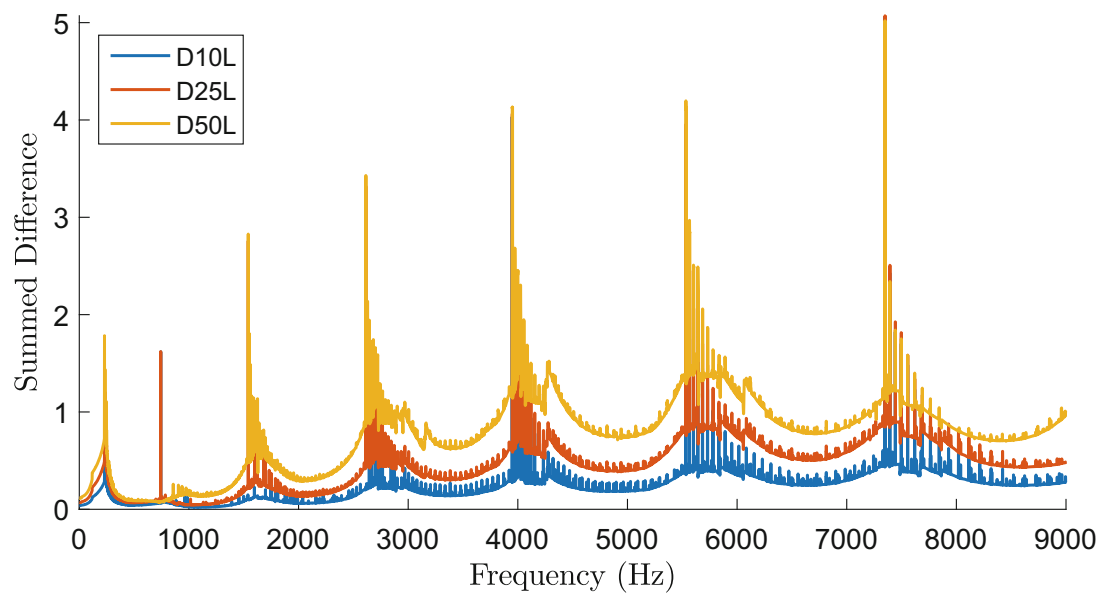


Fig. 21 Test set-up

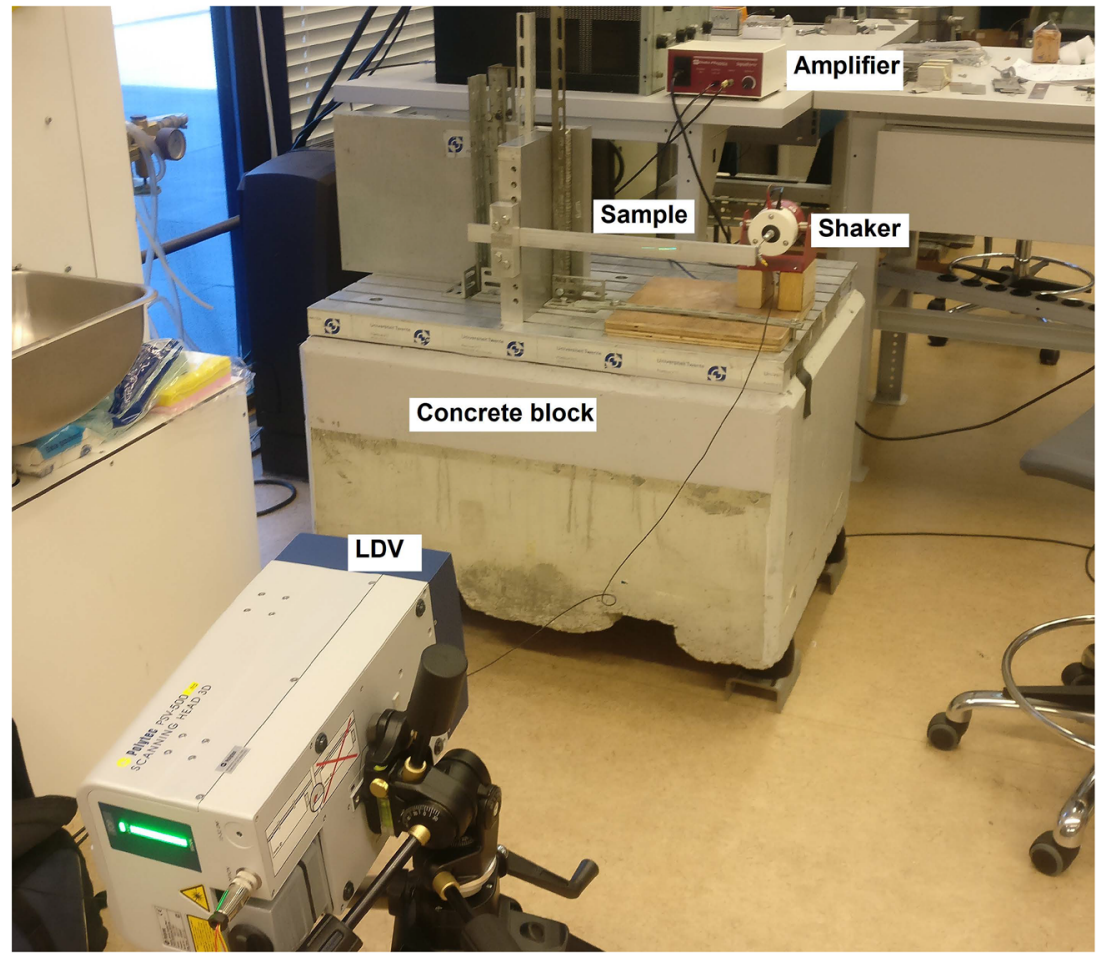

Fig. 22 FRF measurement of the pristine sample

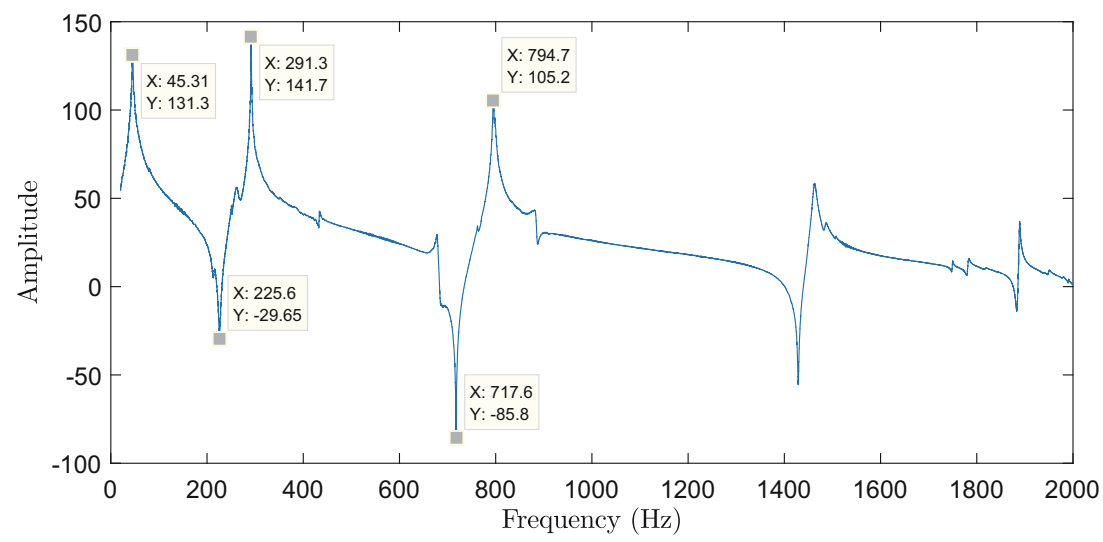

Fig. 23 RASTAR of the pristine case using two different LDV scan rates, $1.1 \mathrm{~Hz}$ and $0.9 \mathrm{~Hz}$

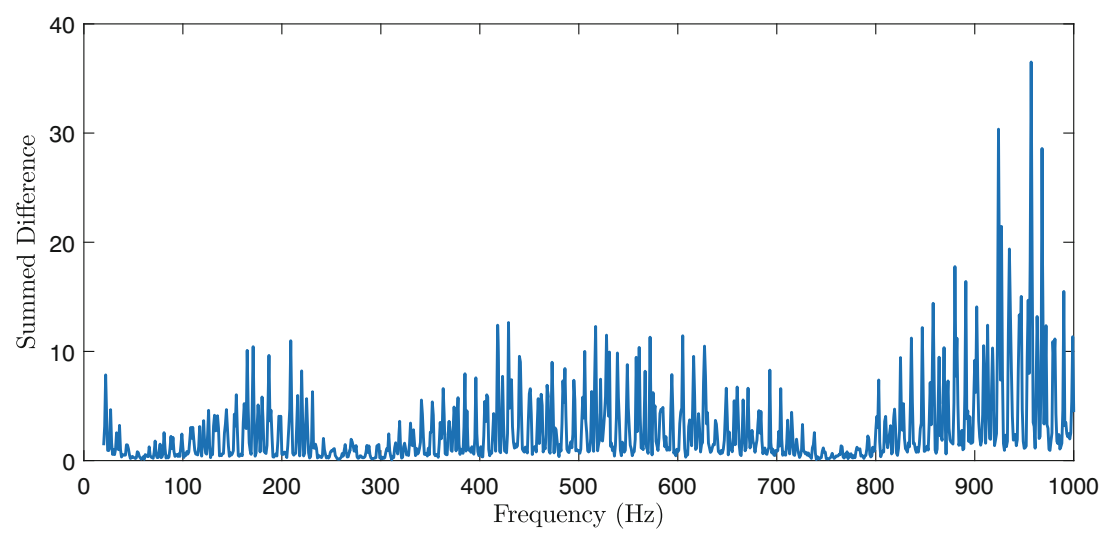


Fig. 24 RASTAR results from unfiltered data

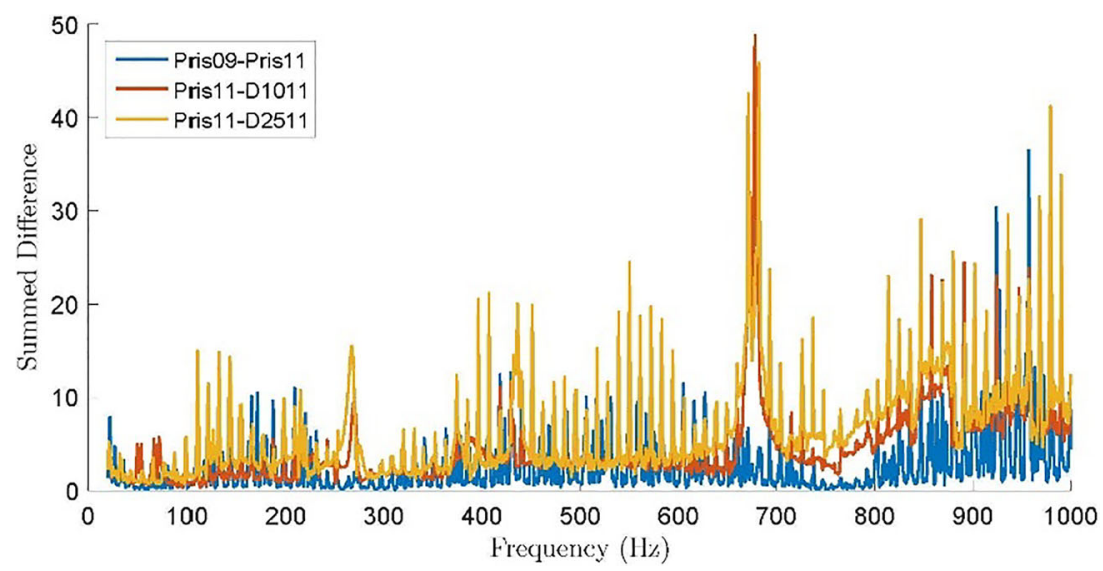

misleading if those are observed by excluding a broader overview of the spectrum. By observing the general trend across the severity levels of the damage, one can appreciate how RASTAR can indicate the presence of structural damage. It is out of a discussion that such a method needs further im- provement in noise reduction to return a much more unequivocably structural condition.
Fig. 25 FFT of indicator graph for the $1 \mathrm{~mm}$ damage case
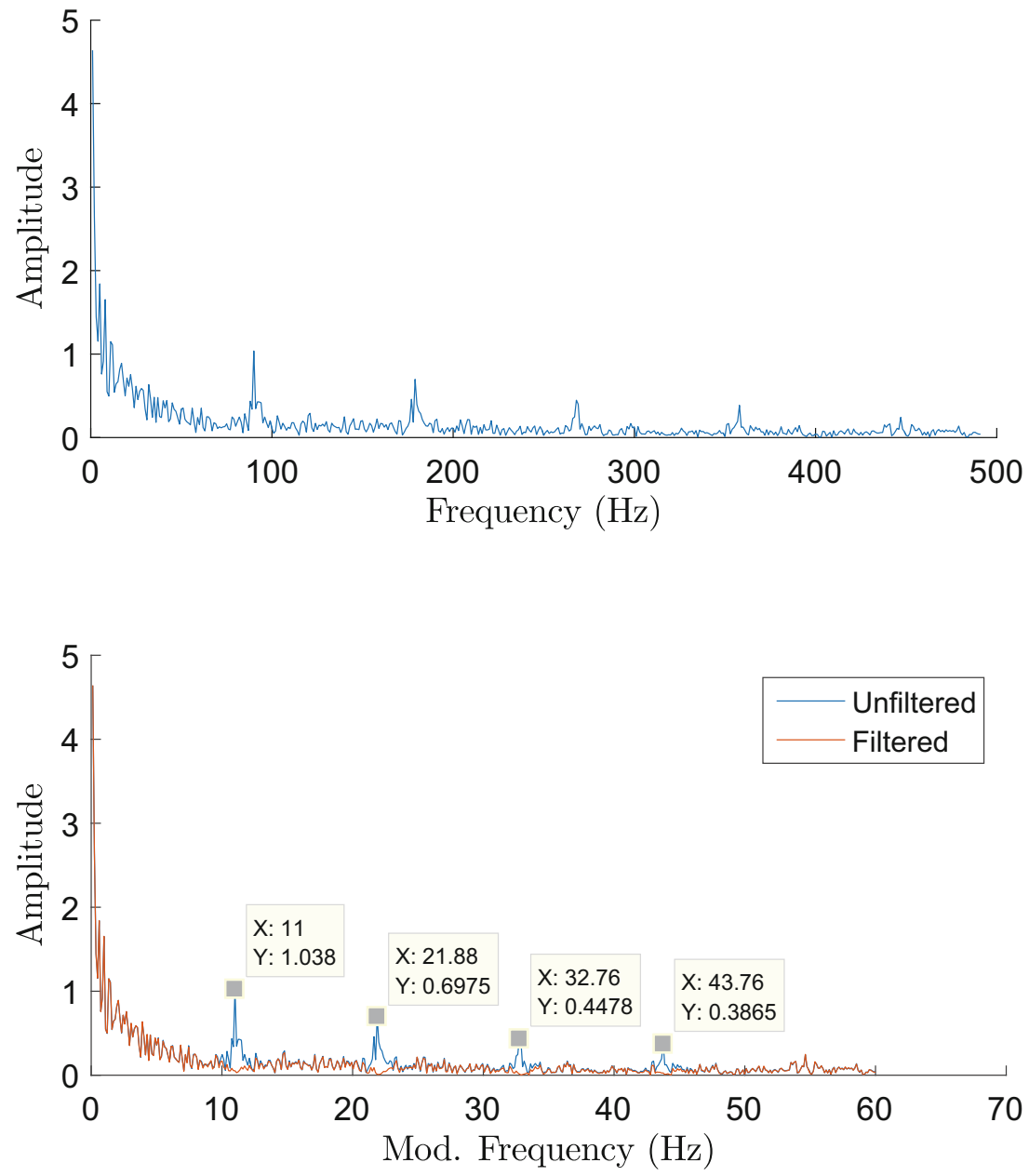

Fig. 26 Filter effect of FFT of $1 \mathrm{~mm}$ damage case with modified $\mathrm{x}$-axis 
Fig. 27 Filtered RASTAR data executed at LDV scan rate of $1.1 \mathrm{~Hz}$

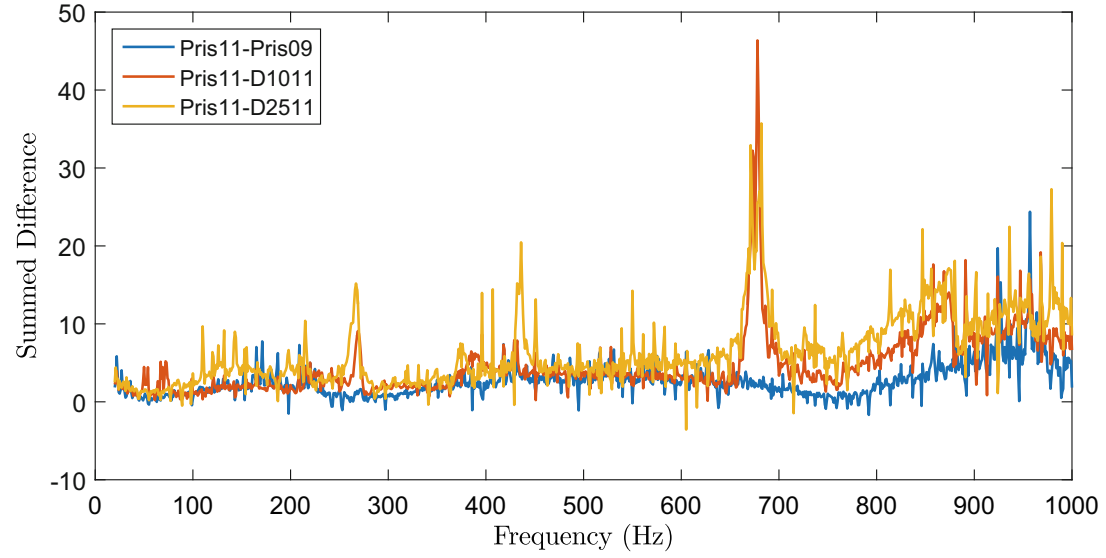

\section{Conclusions}

This research work shows how to perform diagnostics by using continuous scanning methods. The objective was to evaluate the structural integrity by inspection of spectral sidebands, and thus by-passing any deflection shape recovery typically performed with continuous scanning methods. The work was carried out with the aid of a Finite Element Model of a cantilever beam which featured various damage severities and shapes. The simulated FEM ODS were curve-fitted and
Fig. 28 Filtered RASTAR data executed at LDV scan rate of $0.9 \mathrm{~Hz}$

Fig. 29 RASTAR data with experimental FRF
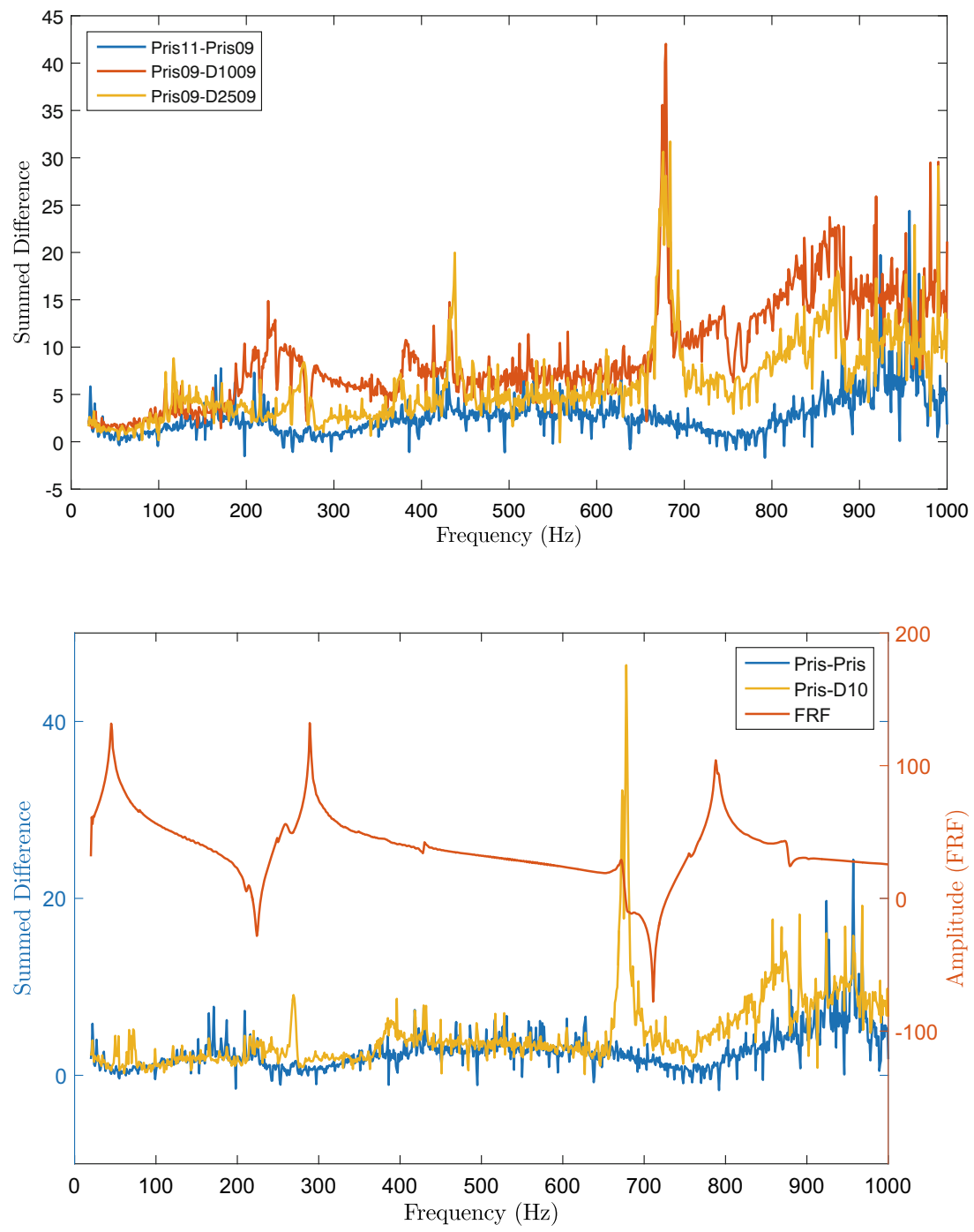
Table 7 Comparison of natural frequencies between the numerical model and experimental test

\begin{tabular}{lll}
\hline$\omega$ & Analytical $(\mathrm{Hz})$ & Experimental $(\mathrm{Hz})$ \\
\hline 1 & 51 & 45 \\
2 & 319 & 291 \\
3 & 889 & 795 \\
\hline
\end{tabular}

the polynomial coefficients used for the simulation of the LDV time series. Several algorithms were developed to analyse the sidebands from pristine and damaged cases, and the RASTAR method proved to be the most effective and reliable one. It takes the relative change of sidebands amplitude, between pristine and damaged cases, over the total summation of the spectral sidebands. RASTAR performed well for the simulated cases where the underlying difference between pristine and damaged case could be observed in several frequency ranges. The experimental results confirmed the performance of the damage indicator, showing clear differences between a pristine and damage conditions from damage as small as $10 \%$ of thickness reduction. The experiments also identified several interesting challenges which could not be simulated, as the change of test conditions when the beam had to be machined for damage allocation. RASTAR showed different magnitudes to the damage severity when one LDV scan rate $(1.1 \mathrm{~Hz})$ was used instead of another one $(0.9 \mathrm{~Hz})$. Nevertheless, the RASTAR performances were not undermined because the indicator can show the difference of structural integrity and proved to be robust to noise. Finally, the structural integrity of, for instance, wind turbine blades can be evaluated over time, or periods of duty cycles, by simply continuously scanning a line and observe the spectral sidebands changes by the RASTAR method. Currently, the method can be exploited for damage detection, but future investigations will extend the RASTAR capabilities to damage identification and locations.

\section{Compliance with Ethical Standards}

Conflict of Interest The Authors do not have any conflict of interest with third party.

Open Access This article is licensed under a Creative Commons Attribution 4.0 International License, which permits use, sharing, adaptation, distribution and reproduction in any medium or format, as long as you give appropriate credit to the original author(s) and the source, provide a link to the Creative Commons licence, and indicate if changes were made. The images or other third party material in this article are included in the article's Creative Commons licence, unless indicated otherwise in a credit line to the material. If material is not included in the article's Creative Commons licence and your intended use is not permitted by statutory regulation or exceeds the permitted use, you will need to obtain permission directly from the copyright holder. To view a copy of this licence, visit http://creativecommons.org/licenses/by/4.0/.

\section{References}

1. Chopra I (2002) Review of state of art of smart structures and integrated systems. AIAA J 40(11):2145-2187

2. Johnson TJ, Brown RL, Adams DE, Schiefer M (2004) Distributed structural health monitoring with a smart sensor array. Mech Syst Signal Process 18(3):555-572

3. Sierra-Pérez J, Torres-Arredondo MA, Güemes A (2016) Damage and nonlinearities detection in wind turbine blades based on strain field pattern recognition. FBGs, OBR and strain gauges comparison. Compos Struct 135:156-166

4. Abry JC, Bochard S, Chateauminois A, Salvia M, Giraud G (1999) In situ detection of damage in CFRP laminates by electrical resistance measurements. Compos Sci Technol 59(6):925-935

5. McCormick N, Lord J (2010) Digital image correlation. Mater Today 13(12):52-54

6. Avril S et al (2008) Overview of identification methods of mechanical parameters based on full-field measurements. Exp. Mech. 48(4):381

7. Mian A, Han X, Islam S, Newaz G (2004) Fatigue damage detection in graphite/epoxy composites using sonic infrared imaging technique. Compos Sci Technol 64(5):657-666

8. Pastor ML, Balandraud X, Grédiac M, Robert JL (2008) Applying infrared thermography to study the heating of 2024-T3 aluminium specimens under fatigue loading. Infrared Phys Technol 51(6):505515

9. Jacquot P (2008) Speckle interferometry: a review of the principal methods in use for experimental mechanics applications. Strain 44(1):57-69

10. Guntaka SR, Manfred H, Torsten F, Ralf U (1994) Speckle interferometry for detection of subsurface damage in fibre-reinforced composites speckle interferometry for detection of subsurface damage in fibre-reinforced composites. Meas Sci Technol 5:100-104

11. De la Torre IM, del Socorro Hernandez Montes M, Flores-Moreno JM, Santoyo FM (2016) Laser speckle based digital optical methods in structural mechanics: A review. Opt Lasers Eng 87: $32-58$

12. Castellini P, Martarelli M, Tomasini EP (2006) Laser Doppler Vibrometry: development of advanced solutions answering to technology's needs. Mech Syst Signal Process 20(6):1265-1285

13. Stanbridge AB, Ewins DJ (Mar. 1999) Modal testing using a scanning laser DOPPLER VIBROMETER. Mech Syst Signal Process 13(2):255-270. https://doi.org/10.1006/mssp.1998.1209

14. Martarelli M (2001) Exploiting the laser scanning facility for vibration measurements," Imperial College of Science, Technology $\{\&\}$ Medicine University of London Expl

15. Chen D-M, Xu YF, Zhu WD (2018) Non-model-based multiple damage identification of beams by a continuously scanning laser Doppler vibrometer system. Measurement 115:185-196

16. Chen D-M, Xu YF, Zhu WD (2018) Identification of damage in plates using full-field measurement with a continuously scanning laser Doppler vibrometer system. J Sound Vib 422:542-567

17. Rothberg SJ et al. 2017 An international review of laser Doppler vibrometry: Making light work of vibration measurement. Opt. Lasers Eng 99, https://doi.org/10.1016/j.optlaseng.2016.10.023

18. Huang Z, Zang C (2019) Damage Detection Using Modal Rotational Mode Shapes Obtained with a Uniform Rate CSLDV Measurement. Appl. Sci. 9(23):4982

19. Huang Z, Zang C (2020) A fast modal rotation measurement using a dual sinusoidal-scan continuously scanning laser doppler vibrometer," Meas Sci Technol

20. Chen D-M, Xu YF, Zhu WD (2018) Non-model-based identification of delamination in laminated composite plates using a continuously scanning laser Doppler vibrometer system. J Vib Acoust $140(4)$ 
21. Chen D-M, Zhu WD (2019) Rapid and Dense 3D Vibration Measurement by Three Continuously Scanning Laser Doppler Vibrometers In: Rotating Machinery, Vibro-Acoustics \& Laser Vibrometry, vol 7, Springer, pp 19-29

22. Chen D-M, Xu YF, Zhu WD (2019) A Comprehensive Study on Detection of Hidden Delamination Damage in a Composite Plate Using Curvatures of Operating Deflection Shapes. J. Nondestruct. Eval. 38(2):54

23. Xu YF, Chen D-M, Zhu WD (2017) Damage identification of beam structures using free response shapes obtained by use of a continuously scanning laser Doppler vibrometer system. Mech Syst Signal Process 92:226-247

24. Khan AZ, Stanbridge AB, Ewins DJ (1999) Detecting damage in vibrating structures with a scanning LDV. Opt Lasers Eng 32(6): 583-592

25. Chiariotti P, Revel GM, Martarelli M (2015) Exploiting Continuous Scanning Laser Doppler Vibrometry and Wavelet Processing for Damage Detection In: Experimental Techniques, Rotating Machinery, and Acoustics, vol 8, Springer, pp 189-196

26. Di Maio D (2016) Damage monitoring using continuous scanning LDV methods: numerical approach. In: 6th International Conference on Optical Measurement Techniques for Structures and Systems III (OPTIMESS2015), Antwerp, pp 61-71
27. Di Maio D (2016) Use of continuous scanning LDV for diagnostics," in International modal analysis conference (IMAC)

28. Rucevskis S, Wesolowski M (2010) Identification of damage in a beam structure by using mode shape curvature squares. Shock Vib 17(4-5):601-610

29. Ramesh L, Rao PS, Kumar KCK, and Prasad DK (2016) Experimental and Finite Element Model Analysis of an uncracked and cracked Cantilever beam. Structure 3(1)

30. Allemang RJ (2003) The modal assurance criterion-twenty years of use and abuse. Sound Vib 37(8):14-23

31. Stanbridge AB, Ewins DJ (1999) modal testing using a scanning laser doppler vibrometer AB Stanbridge \& DJ Ewins Imperial College of Science Technology and Medicine, exhibition road, London, SW7 2BX, U.K.," Mech. Syst. Signal Process

32. Bruinsma S, Di Maio D, Tinga T (2018) Diagnostics based on continuous scanning LDV methods: numerical study. Journal of physics: Conference series 1149(1):12012

33. Martarelli M, Ewins DJ (2006) Continuous scanning laser Doppler vibrometry and speckle noise occurrence. Mech Syst Signal Process 20(8):2277-2289

Publisher's Note Springer Nature remains neutral with regard to jurisdictional claims in published maps and institutional affiliations. 